\title{
The T Tauri star RY Tauri as a case study of the inner regions of circumstellar dust disks ${ }^{\star}$
}

\author{
A. A. Schegerer ${ }^{1}$, S. Wolf ${ }^{1}$, Th. Ratzka ${ }^{2}$, and Ch. Leinert ${ }^{1}$ \\ 1 Max-Planck-Institut für Astronomie, Königstuhl 17, 69117 Heidelberg, Germany \\ e-mail: schegerer@mpia-hd.mpg.de \\ 2 Astrophysikalisches Institut Potsdam, An der Sternwarte 16, 14482 Potsdam, Germany
}

Received 3 January 2007 / Accepted 13 November 2007

\section{ABSTRACT}

\begin{abstract}
Aims. We study the inner region ( 1.0 AU up to a few 10 AUs) of the circumstellar disk around the "classical" T Tauri star RY Tau. Our aim is to find a physical description satisfying the available interferometric data, obtained with the mid-infrared interferometric instrument at the Very Large Telescope Interferometer, as well as the spectral energy distribution in the visible to millimeter wavelength range. We also compare the findings with the results of similar studies, including those of intermediate-mass $\mathrm{Herbig} \mathrm{Ae} / \mathrm{Be}$ stars.

Methods. Our analysis is done within the framework of a passively heated circumstellar disk, which is optionally supplemented by the effects of accretion and an added envelope. To achieve a more consistent and realistic model, we used our continuum transfer code MC3D. In addition, we studied the shape of the $10 \mu \mathrm{m}$ silicate emission feature in terms of the underlying dust population, both for single-dish and for interferometric measurements.

Results. We show that a modestly flaring disk model with accretion can explain both the observed spectral energy distribution and the mid-infrared visibilities obtained with the mid-infrared infrared instrument. We found an interesting ambiguity: a circumstellar active disk model with an added envelope, and a lower accretion rate than in the active disk model without envelope, could represent the observations equally as well. This type of model with the envelope should be considered a viable alternative in future models of other T Tauri stars. The approach of a disk with a puffed-up inner rim wall and the influence of a stellar companion is also discussed. We also investigate the influence of various fit parameters on the outcome of the radiative transfer modeling. From the study of the silicate emission feature we see evidence for dust evolution in a T Tauri star, with a decreasing fraction of small amorphous and an increasing fraction of crystalline particles closer to the star.
\end{abstract}

Key words. infrared: stars - accretion, accretion disks - astrochemistry - stars: planetary systems: protoplanetary disks radiative transfer - instrumentation: interferometers

\section{Introduction}

T Tauri stars are known as precursors of low-mass main sequence stars ( $\left.\leqq 2-3 M_{\odot}\right)$. In contrast to main sequence stars, their characteristic properties are strong emission line radiation (e.g., Balmer $\alpha$ ) and excessive continuum radiation observed in the $\mathrm{UV}$, infrared and the millimeter ( $\mathrm{mm}$ ) wavelength range of their spectral energy distribution (SED). It has been shown that the spatial distribution of circumstellar dust in a disk or an envelope that is primarily exposed to stellar radiation, is responsible for the excess radiation in the infrared wavelength range (e.g., Adams et al. 1987), while accretion of circumstellar material results in the UV excess and strong emission line radiation (see Hartmann 1998, for a review).

The extraordinary interest in the inner region of a circumstellar disk results from the assumption that the formation of planets is favored there (see Nagasawa et al. 2006; Wünsch et al. 2005; Klahr 2004). While mm observations probe cooler outer disk regions and layers close to the midplane of circumstellar disks, observations in the mid-infrared (MIR) wavelength regime are more sensitive to warmer $(250 \mathrm{~K} \leqq T \leqq 1000 \mathrm{~K}$; see Schegerer et al. 2006: Fig. 1) disk regions, such as the surface of the inner regions where dust is directly irradiated by the central star.

^ Appendix A and Fig. A1 are only available in electronic form at http://www. aanda.org
However, studies of the inner circumstellar regions ( $\sim$ AU) of objects in the closest star forming regions are only feasible by interferometric observations.

Strong emission features in the MIR range at $10 \mu \mathrm{m}$ and $20 \mu \mathrm{m}$ corresponding to the $\mathrm{Si}-\mathrm{O}$ stretching and bending modes of silicate grains, are assumed to result from absorption and reemission processes in optically thin dust layers of the circumstellar disks. While silicate grains are expected to be initially amorphous and small $(\leqq 0.1 \mu \mathrm{m}$; Mathis et al. 1977, MRN thereafter $)^{1}$, the crystallization of amorphous silicates starts at temperatures of $\sim 1200 \mathrm{~K}$ (e.g., Gail 1998). Moreover, high dust densities and turbulent processes in the interior of circumstellar disks favor dust grain growth to dust pebbles (e.g., Blum et al. 2000; Johansen et al. 2005). The shape of the emitted silicate feature allows the estimation of the predominant stage of the dust evolution in a young stellar object (YSO). Different degrees of crystallization and grain growth have already been shown in a large sample of T Tauri stars of different ages and stellar masses (e.g., Schegerer et al. 2006). As temperature and density increase in circumstellar disks with decreasing distance to the central star, crystallinity and grain size sensitively depend on the radial position of the dust in a circumstellar system (e.g.,

\footnotetext{
1 In this paper amorphous and small, i.e., primordial and interstellar dust grains, are called not-evolved/undeveloped.
} 


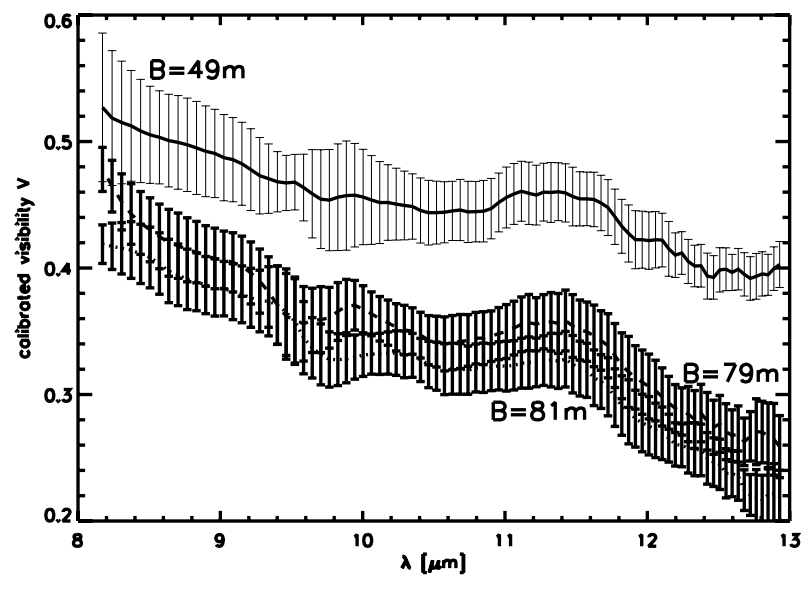

Fig. 1. The spectrally resolved, calibrated visibility data derived from our MIDI observations (Table 3). The error bars are the $1 \sigma$ deviations that result from the observations of different calibrators in one night.

Beckwith et al. 1999; Weidenschilling 1997; Gail 2003: Fig. 28). In fact, observations with MID-infrared Interferometric instrument (MIDI) have already revealed a correlation between the radial position and the evolutionary stage of silicate dust in circumstellar disks around Herbig $\mathrm{Ae} / \mathrm{Be}(\mathrm{HAeBe})$ stars, which are the more massive counterparts of T Tauri stars (Leinert et al. 2004; van Boekel et al. 2004).

The density, temperature and compositional structure of circumstellar dust disks and surrounding envelopes have been the central issue of many former studies (e.g., Chiang \& Goldreich 1997; D’Alessio et al. 2001). Different modeling approaches have been tried to quantitatively explain and reproduce phenomenons like excess radiation, shapes of emission lines (e.g., Muzerolle et al. 2004; Natta et al. 2000), flux variations (e.g., Herbig et al. 2003) and intensity distributions of images (e.g., Lucas et al. 1997). However, the evolution of (inner) disk structure and its correlation to dust evolution is still unclear (e.g., Millan-Gabet et al. 2006; Beckwith et al. 1999), and has been underestimated in actual modeling approaches, or mainly reserved for theoretical studies (e.g., Gail 1998). However, interferometric observations in the MIR wavelength range, which are now available, are sensitive to the inner disk structure where warm dust dominates. Including their sensitivity for the silicate feature, the correlation between inner disk structure and grain evolution can be directly studied.

In this paper we focus on modeling of the SED and spectrally resolved $\mathrm{N}$ band visibilities, which we obtained for the T Tauri star RY Tau with MIDI. The key questions of this study are the following: Is it possible to simultaneously model the SED and N band visibilities of RY Tau solely by an externally, i.e., passively heated, disk? Do different extensions of this model reproduce the observations, simultaneously? What do we learn about the (silicate) dust composition of the disk?

The result of previous measurements of RY Tau are presented in Sect. 2. In Sect. 3 we outline the observations of RY Tau and the subsequent data reduction. We present the radiative transfer code and the basic dust set of our modeling approach in Sect. 4. In the following Sect. 5 we compare the results of the different modeling approaches we used, i.e., the ative disk model with and without an envelope, and point to supplements. In Sect. 6 the dust composition of the upper disk layers and its dependence on the radial distance from the central star is studied. Finally, in Sect. 7 we draw comparisons between the used models, refer to previous results, including the
Table 1. Observed properties of RY Tau.

\begin{tabular}{llr}
\hline \hline Parameter & Value & Reference \\
\hline RA (J2000.0) & 042157.4 & 1 \\
DEC (J2000.0) & +282636 & 1 \\
Distance & $134_{-31}^{+54} \mathrm{pc}$ & 2 \\
Visual extinction & $(2.2 \pm 0.2) \mathrm{mag}$ & 3 \\
Spectral type & F8 III & 4 \\
Stellar mass & $1.69 M_{\odot}$ & 5 \\
Stellar luminosity & $12.8 L_{\odot}$ & 6 \\
Accretion rate & $7.8 \times 10^{-8} M_{\odot} / \mathrm{yr}$ & 3 \\
Age & $(6.5 \pm 0.9) \mathrm{Myr}$ & 7 \\
\hline
\end{tabular}

References - 1: Perryman et al. (1997); 2: Bertout et al. (1999); 3: Calvet et al. (2004); 4: Mora et al. (2001); 5: Beckwith et al. (1990); 6: Akeson et al. (2005); 7: Siess et al. (1999).

Table 2. Photometric measurements of RY Tau. The data of different measurements are averaged and the standard deviation is determined. For convience, all fluxes are given in Jansky and in magnitude. Conversion factors are taken from Leinert (1997).

\begin{tabular}{llr}
\hline \hline Wavelength $[\mu \mathrm{m}]$ & Flux [Jy] $([\mathrm{mag}])$ & Reference \\
\hline $0.36(U)$ & $0.04 \pm 0.01(11.72 \pm 0.16)$ & 1 \\
$0.45(B)$ & $0.14 \pm 0.01(11.23 \pm 0.08)$ & 1 \\
$0.55(V)$ & $0.33 \pm 0.01(10.12 \pm 0.04)$ & 1 \\
$0.64\left(R_{\mathrm{C}}\right)$ & $0.59 \pm 0.03(9.3 \pm 0.05)$ & 1 \\
$0.79\left(I_{\mathrm{C}}\right)$ & $1.0 \pm 0.06(8.52 \pm 0.06)$ & 1 \\
$1.25(J)$ & $1.6 \pm 0.8(7.64 \pm 0.40)$ & 2,3 \\
$1.65(H)$ & $2.3 \pm 0.9(6.48 \pm 0.30)$ & 2,3 \\
$2.20(K)$ & $3.8 \pm 0.5(5.56 \pm 0.13)$ & 2,3 \\
$4.80(M)$ & $6.6 \pm 2.0(3.4 \pm 0.28)$ & 4,5 \\
$11.0(N)$ & $20.0 \pm 0.3$ & 6 \\
25 & $28 \pm 3$ & 7 \\
60 & $18 \pm 4$ & 7,8 \\
100 & $12 \pm 5$ & 9 \\
1300 & $0.23 \pm 0.02$ & \\
\hline
\end{tabular}

References - 1: Calvet et al. (1997); 2: Gezari-Catalog (Gezari et al. 1999); 3: 2 MASS-Catalog (Cutri et al. 2003); 4: Rydgren et al. (1976); 5: Elias (1978); 6: Hanner et al. (1998); 7: Strom et al. (1989); 8: Harvey et al. (1979); 9: Mora et al. (2001).

models of Akeson et al. (2005) where near-infared (NIR) visibilites were modeled, and discuss discrepancies. Furthermore, we investigate the possibility of the existence of a stellar companion and compare RY Tau with HAeBe stars. Section 8 summarizes our results.

\section{Previous measurements}

RY Tau, demonstrably observed for the first time in 1907 (Pickering 1907), is a well-known T Tauri star (Joy 1945) that belongs to the Taurus-Auriga molecular cloud at a distance of $\sim 140$ pc. Table 1 shows the main properties of this object, which are obtained from previous measurements. Photometric fluxes are listed in Table 2.

RY Tau is a UX Ori-type star, i.e., this T Tauri object has revealed irregular photometric variability in the visible and NIR wavelength range. During several months in 1983/84 and in $1996 / 97$, its visible brightness increased from $\sim 11$ th to $\sim 9$ th magnitude and decreased again to its initial value (Herbst \& Stine 1984; Zajtseva et al. 1985; Petrov et al. 1999; Herbst \& Shevchenko 1999). Such a rare but strong variability is conventionally explained by variations of the obscuration of the central star caused by an inclined circumstellar disk and an envelope (e.g. Eiroa et al. 2002). Smaller variations $(\Delta V \approx 0.1, \Delta J \approx 0.2$, 
Table 3. Summary of the MIDI observations of RY Tau and calibrators. The dates, UT, $L$ (in $\mathrm{m}$ ) and PA (in degrees, measured from North to East) of the sky-projected baselines are listed. The airmass $A M$ in the right column is given for the time of fringe tracking. The observations with a projected baseline of $79 \mathrm{~m}$ and $81 \mathrm{~m}$ provided an almost identical result (see Fig. 1).

\begin{tabular}{cccccc}
\hline \hline Date & UT & Object & $L$ & PA & $A M$ \\
\hline Nov. 1st, 2004 & $3: 54-4: 16$ & HD 25604 & 74 & 96 & 1,68 \\
Nov. 1st, 2004 & $4: 37-4: 56$ & RY Tau & 79 & 97 & 1.83 \\
Nov. 1st, 2004 & $4: 58-5: 07$ & RY Tau & 81 & 95 & 1.79 \\
Nov. 1st, 2004 & $5: 54-6: 10$ & HD 49161 & 64 & 87 & 1.56 \\
Nov. 1st, 2004 & $7: 03-7: 17$ & HD 31421 & 89 & 82 & 1.28 \\
& & & & & \\
Nov. 4th, 2004 & $0: 01-0: 26$ & HD 178345 & 57 & 146 & 1.42 \\
Nov. 4th, 2004 & $2: 19-2: 47$ & HD 188603 & 46 & 169 & 2.47 \\
Nov. 4th, 2004 & $3: 16-3: 54$ & HD 25604 & 61 & 117 & 1.75 \\
Nov. 4th, 2004 & $5: 11-5: 29$ & HD 20644 & 59 & 102 & 1.69 \\
Nov. 4th, 2004 & $7: 18-7: 36$ & HD 37160 & 61 & 107 & 1.21 \\
Nov. 4th, 2004 & $7: 44-8: 00$ & RY Tau & 49 & 92 & 1.95 \\
Nov. 4th, 2004 & $9: 00-9: 23$ & HD 50778 & 61 & 113 & 1.04 \\
\hline
\end{tabular}

$\Delta K \approx 0.2$ ) in the range of several days were also detected (Eiroa et al. 2002). By a comparison between the maximum and minimum brightness of the object the photometric measurements, listed in Table 2, and our observations with MIDI correspond to the "quiescent" state of the object, i.e., close to the photometric minimum.

There is a wide range of values measured for the visual extinction $A_{\mathrm{V}}$ of RY Tau (Kuhi 1974: $1.3 \mathrm{mag}$; Cohen \&

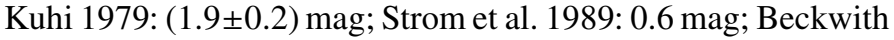
et al. 1990: $2.7 \mathrm{mag}$; Kenyon \& Hartmann 1995: $1.8 \mathrm{mag})$. We adopt a value recently derived by Calvet et al. (1997): $A_{\mathrm{V}}=$ $(2.2 \pm 0.2) \mathrm{mag}$. The level of veiling in the visible range of the spectrum is low $(\geqq 0.1$; Basri et al. 1991; Hartigan et al. 1995; Petrov et al. 1999) but markedly higher in the infrared range (>0.8; Folha \& Emerson 1999).

A potential duplicity/multiplicity was not found by Leinert et al. (1993) by using NIR speckle interferometry reaching a spatial resolution between $0.13^{\prime \prime}$ and $13^{\prime \prime}$ but the regular variation of the photocenter, found by the astrometric measurements of HIPPARCOS, could be a hint for a companion with a projected minimum distance of $3.27 \mathrm{AU}$ (23.6 mas) and a position angle of $304^{\circ} \pm 34^{\circ}$ (Bertout et al. 1999).

\section{Interferometric observations and data reduction}

\subsection{Observing sequence}

RY Tau was observed with MIDI/VLTI (Very Large Telescope Interferometer; Leinert et al. 2003a) in 2004, November 1st and 4 th, within the scope of guaranteed time observations. The dates and universal times (UT) of the observations, as well as skyprojected baseline lengths (L) and position angles (PA) of the interferometer, are listed in Table 3. An observing sequence consists of the following steps:

i. Single-telescope imaging is used for a highly precise acquisition of the object within a field-of-view of $2 \times 2^{\prime \prime}$ in order to guarantee a maximum overlap of the telescope beams.

ii. After the beam combiner is introduced into the optical path, the characteristic interference pattern (fringe pattern) is found around an optical path difference (OPD) of zero. A low resolution prism $(\lambda / \delta \lambda \approx 30)$, which is put in the optical path of the combined beams, allows us to obtain spectrally resolved interferograms and the wavelength-dependent correlated flux $F_{\text {corr }}(\lambda)$.

iii. The spectrum $F_{\text {total }}(\lambda)$ is determined by single-telescope exposures that are recorded on the same detector pixels as the fringe signal $F_{\text {corr }}(\lambda)$. By definition, the spectrally resolved visibility $V(\lambda)$ is obtained as the ratio of the correlated and the total flux

$$
V(\lambda)=\frac{F_{\text {corr }}(\lambda)}{F_{\text {total }}(\lambda)}
$$

This visibility is still biased by instrumental effects.

iv. The transfer function of the instrument is determined by the observation of calibrator stars before and after the observation of the scientific target. A known transfer function is required for the elimination of the instrumental and atmospheric influences. The calibrators are selected for their known diameter, the absence of strong photometric variabilities and companions, and their angular distance to the scientific target. Some of the calibrators were also used for absolute flux calibration. The error of our calibrated visibility $V(\lambda)$ is the $1 \sigma$ deviation that is obtained by the observations of different calibrators in one night (see Table 3).

Instrumental informations and observing procedures are described by Leinert et al. (2003b,c, 2004) and Ratzka (2005).

\subsection{Data reduction}

The reduction procedure of MIDI data is complex and has been described in detail by Leinert et al. (2004), Ratzka (2005), and Jaffe (2004). The data obtained with MIDI were reduced with the MIA software that is based on power spectrum analysis and the results were independently confirmed by using the EWS software. The EWS software contains a coherent integration algorithm, which involves a kind of shift-and-add in the complex plane. Both reduction software packages are publicly available ${ }^{2}$.

\subsection{Observational results}

The resulting wavelength dependent visibility curves for the three baselines are shown in Fig. 1, including $1 \sigma$ error bars. The spectrophotometry of the silicate emission band $F_{\text {total }}(\lambda)$, obtained during the measurements, is shown in Fig. 7, and also included in the SED of RY Tau (Fig. 6). Figure 7 also shows the observed correlated fluxes $F_{\text {corr }}(\lambda)$, formally obtained as a product of these two quantities (Eq. (1)). We refer to the nearcoincidence of the observations at baseline lengths of $79 \mathrm{~m}$ and $81 \mathrm{~m}$.

\section{Tools}

\subsection{MC3D - Monte Carlo code for radiative transfer}

In contrast to many previous investigations where the radiative transfer function has been solved (e.g., Sonnhalter et al. 1995; Chiang \& Goldreich 1997; Dullemond et al. 2001), we use the well-tested code MC3D, which is based on the Monte-Carlo method (Wolf et al. 1999; Pascucci et al. 2004). Considering an axially symmetric object, we assume a two-dimensional geometry in a polar coordinate system $(r, \theta)$ with a logarithmic grid spacing in $r$ and a uniform grid spacing in $\theta$. Heating sources like

\footnotetext{
2 http://wWw.mpia-hd.mpg.de/MIDISOFT/ and http://www.strw. leidenuniv.nl/ koehler/MIA+EWS-Manual/
} 
the central star, accretion effects, and heated dust grains determine the temperature distribution. The flux of the central star is determined by the theoretical stellar atmosphere model provided by the Kurucz (1994). The product of the dust-specific absorption efficiency $Q_{\text {abs }}(\lambda)$, the grain surface and the blackbody emission $B_{v}\left(T_{\text {dust }}\right)$ represent the flux that a dust grain with a temperature of $T_{\text {dust }}$ reemits in local thermal equilibrium (Kirchhoff's law of local thermal equilibrium). Sources like the central star and accretion are treated as blackbody emitters. Gas molecules and atoms are not considered in our models.

The radial density distribution of the disk is given by the surface density profile

$\Sigma(r)=\Sigma_{0} \cdot r^{-p}$

with the radial coordinate $r$, an exponent $p$ and a constant $\Sigma_{0}=100 \mathrm{~g} \mathrm{~cm}^{-2}$ (Weidenschilling 1977). The vertical density distribution is calculated self-consistently assuming hydrostatic equilibrium, i.e., the balance of gravitational and thermal pressure (Schegerer et al., in prep.).

After temperature and density distribution have been determined, the SED and the projected image of the star and its circumstellar environment, considering an inclination angle $\vartheta$, are calculated. The resolution of the image is by a factor of $\sim 10$ better than the resolution of the observations. For projected baselines of $79 \mathrm{~m}$ and $49 \mathrm{~m}$, our observations with MIDI reached spatial resolutions of $\sim 1.8 \mathrm{AU}$ and $\sim 2.8 \mathrm{AU}$ at a distance of $\sim 140 \mathrm{pc}^{3}$.

\subsection{Dust model}

The infrared excess that is emitted from YSOs originates from heated dust in the circumstellar environment. Assuming compact, homogeneous, and spherical dust grains, their optical properties, such as scattering and extinction cross sections, are determined by Mie scattering-theory from the measured complex refractive index of the specific material (Bohren \& Huffman 1983). In our modeling approach, we assume a dust mixture of "astronomical silicate" and graphite with the relative abundances of $62.5 \%$ for astronomical silicate and $37.5 \%$ for graphite (Draine \& Malhotra 1993). The dielectric function of astronomical silicate was formerly synthesized by Draine \& Lee (1984) in order to reproduce the extinction of different silicate compounds in interstellar space. We consider an improved version of this dielectric function (Weingartner \& Draine 2001) ${ }^{4}$, which was recently confirmed by a study of the interstellar extinction in the NIR wavelength range (Indebetouw et al. 2005). For graphite we adopt the $\frac{1}{3}: \frac{2}{3}$ ratio with $\kappa_{v}=\left[\kappa_{v}\left(\epsilon_{\|}\right)+2 \kappa_{v}\left(\epsilon_{\perp}\right)\right] / 3$, where $\epsilon_{\|}$and $\epsilon_{\perp}$ are the components of the graphite dielectric tensor for the electric field parallel and perpendicular to the crystallographic c-axis and $\kappa_{v}$ is the mass absorption coefficient. The strongly absorbing graphite grains efficiently contribute to the heating of the dusty circumstellar environment. The ratio of the extinction efficiency factor of carbon dust to the extinction efficiency factor of silicate dust is $\sim 10$ in the NIR wavelength range (Draine \&Lee 1984; Wolf \& Hillenbrand 2003).

We consider a grain size power law $n(a) \propto a^{-3.5}$ with $a_{\text {min }} \leqq$ $a \leqq a_{\max }$, where $n(a)$ is the number of dust particles with radius $a$. This power law was formerly found by MRN studying extinction of interstellar carbon and silicate with typical sizes between

\footnotetext{
3 The spatial resolution power $R$ of an interferometer is given by the ratio of the observing wavelength $\lambda$ and the sky-projected distance between a telescope pair, i.e., the effective baseline length $B: R=\lambda /(2 B)$.

${ }^{4}$ See http://www . astro.princeton. edu/ draine
}

$0.005-0.01 \mu \mathrm{m}$ and $0.025-0.25 \mu \mathrm{m}$, respectively. This grain size power law has already been used in former modeling aproaches of YSOs. We use a minimum particle size of $a_{\mathrm{min}}=0.005 \mu \mathrm{m}$ in all of our modeling approaches.

The maximum grain size $a_{\max }$ affects the mass absorption coefficient $\kappa_{v}(a)$ of dust, i.e., $\kappa_{v}(a)$ increases with $a_{\max }$ for sizes up to a few mm. The mm flux depends on the disk mass $M_{\text {disk }}$ and the absorption coefficient $\kappa_{v}(a)$, i.e., $a_{\max }$, in particular, when assuming an optically thin disk in the mm wavelength range. Correspondingly, the spectral index $\alpha\left(F_{v} \propto v^{\alpha}\right)$ decreases with an increase of $a_{\max }$ from an absolute value of $\sim 4$ (for $a_{\max }<0.1 \mu \mathrm{m}$ ) to $\sim 2$ (only for large bodies). The correlation between the spectral index $\alpha$ and the maximum dust grain size $a_{\max }$ was formerly studied by D'Alessio et al. (2001), while Wood et al. (2002) also investigated the correlation between disk mass and mm flux.

In our modeling approach, we have found that a dust distribution with a maximum grain size of $a_{\max }=0.25 \mu \mathrm{m}$ and the above mentioned grains size power law for silicate and carbon generally underestimates the mm flux unless a disk mass in the range of $\sim 1.0 M_{\odot}$ is assumed. Circumstellar disks with such high masses are potentially gravitationally unstable (e.g., Laughlin \& Bodenheimer 1994; Boss 2000; Lodato \& Bertin 2001). Moreover, an upper grain size of $a_{\max }=0.25 \mu \mathrm{m}$ results in a too steep mm slope, in contrast to the measured spectral in$\operatorname{dex} \alpha$. Using the Very Large Array for their mm measurements, Rodmann et al. (2006) found a spectral index of $\alpha=2.55 \pm 0.09$ for RY Tau and derived a maximum grain size of $a_{\max }=1 \mathrm{~mm}$. The latter maximum grain size is used in our modeling approach. We have to mention that the spectral index $\alpha$ provides only a lower limit for the maximum dust size as it converges for $a_{\max }>1 \mathrm{~mm}$.

Although the mm wavelength range of the observed SED can sufficiently be simulated considering grain sizes up to $1 \mathrm{~mm}$ and relatively low disk masses $\left(<10^{-2} M_{\odot}\right)$, the spectral contribution in the NIR wavelength range strongly decreases with increasing maximum grain size. The dust particles with $a_{\max }>1 \mu \mathrm{m}$ can be less effectively heated than the smaller particles of the canonical MRN distribution ${ }^{5}$. This effect is the reason why we implement a two-layer dust model in our modeling approach. The disk interior contains a maximum dust grain size of $1 \mathrm{~mm}$ while the MRN grain size distribution with $a_{\max }=0.025 \mu \mathrm{m}$ is used in the upper disk layers where the optical depth $\tau_{\mathrm{N}}$ in $\mathrm{N}$ band, measured vertical to the disk midplane, falls below unity. Such a division of the disk is based on the idea of the favored settling of larger dust grains. Furthermore, dust particles are assumed to mainly grow in the denser regions of the disk close to the midplane (e.g., Lissauer 1993; Blum \& Wurm 2000). Similar disk models with two or more different dust layers have already been proposed by Chiang \& Goldreich (1997) and used by Whitney et al. (2003), for instance. However, it is still an open question how strongly dust grains are mixed in the circumstellar environment (e.g., D’ Alessio et al. 1997; Gail 1998; McCabe et al. 2003; Wolf et al. 2003).

In order not to determine the temperature distribution of each single dust component and to accelerate the radiative transfer simulations, we construct an "artificial" particle with optical constants that are derived by averaging the optical properties of carbon and astronomical silicate of different sizes in each dust layer. Such an approach was justified by Wolf (2003).

5 For all different dust sets we assume a constant exponent of -3.5 for the grain size power law $n(a)$. 
Table 4. Parameter set for the passive disk model, the active model (Sect. 5.1) and the active model with an envelope (Sect. 5.3). Results correspond to Fig. 6.

\begin{tabular}{lrrr}
\hline \hline Parameter & $\begin{array}{r}\text { Passive disk } \\
\text { model }\end{array}$ & $\begin{array}{r}\text { Active disk } \\
\text { model }\end{array}$ & $\begin{array}{r}\text { Active disk + envelope } \\
\text { model }\end{array}$ \\
\hline Stellar mass $M_{\star}$ & $1.69 M_{\odot}$ & $1.69 M_{\odot}$ & $1.69 M_{\odot}$ \\
Stellar temperature $T_{\star}$ & $5560 \mathrm{~K}$ & $5560 \mathrm{~K}$ & $5560 \mathrm{~K}$ \\
Stellar luminosity $L_{\star}$ & $10.0 L_{\odot}$ & $10.0 L_{\odot}$ & $10.0 L_{\odot}$ \\
Disk mass $M_{\text {disk }}$ & $1 \times 10^{-2} M_{\odot}$ & $4 \times 10^{-3} M_{\odot}$ & $4 \times 10^{-3} M_{\odot}$ \\
Outer disk radius $R_{\text {out }}$ & $270 \mathrm{AU}$ & $270 \mathrm{AU}$ & $270 \mathrm{AU}$ \\
Inner disk radius $R_{\text {in }}$ & $0.3 \mathrm{AU}$ & $0.3 \mathrm{AU}$ & $0.3 \mathrm{AU}$ \\
Exponent $p$ (see Eq. (2)) & 1.3 & 1.3 & 1.4 \\
Inclination $\vartheta$ & $\leqq 70^{\circ}$ & $\leqq 70^{\circ}$ & $\leqq 65^{\circ}$ \\
$c_{1}$ (see Eq. (5)) & - & - & $5.0 \times 10^{-5}$ \\
$c_{2}$ (see Eq. (5)) & - & - & 1.0 \\
Accretion rate $\dot{M}$ & - & $9.1 \times 10^{-8} M_{\odot} \mathrm{yr}^{-1}$ & $2.5 \times 10^{-8} M_{\odot} \mathrm{yr}^{-1}$ \\
Boundary temperature $T_{\text {bnd }}$ & - & $8000 \mathrm{~K}$ & $8000 \mathrm{~K}$ \\
Truncation radius $R_{\text {bnd }}$ & - & $5 R_{\star}$ & $5 R_{\star}$ \\
\hline
\end{tabular}

\section{Models of the density structure}

In the following subsections we will present our approaches to model the SED and the MIR visibilities that we obtained with MIDI. An active disk model is our favorite approach. Such a model was also used to reproduce SED and $K$ Band visibilities of RY Tau obtained with the Palomar Testbed Interferometer (Akeson et al. 2005). Additionally, an active disk model with an envelope and potential supplements are discussed. One of the main issues of this study is to clarify if the models we use can be distinguished.

The existence of a passively-heated circumstellar disk in our analysis is beyond all questions. The paradigm that the formation of a disk is one of the evolutionary stages of circumstellar stuctures has been finally confirmed by the images of many YSOs in different wavelength ranges (e.g., Padgett et al. 1999; Mannings \& Sargent 1997, 2000). The T Tauri star RY Tau is a Class II object (Kenyon \& Hartmann 1995) where a surrounding disk has already formed. Therefore, a passively heated disk is a basic ingredient and will be retained in our different modeling approaches.

The disk model is characterized by the disk mass $M_{\text {disk }}{ }^{6}$, the inner $R_{\text {in }}$ and outer disk radius $R_{\text {out }}$, the inclination angle $\vartheta$, and an exponent $p$, which represents the radial dependence of the surface density $\Sigma$ (see Eq. (2)). The inner radius $R_{\text {in }}$ is given in advance, but can also be considered as a starting value that is iterated until the temperature at the inner radius falls below the the sublimation temperature of $1600 \mathrm{~K}$ (Duschl et al. 1996). Properties of the central star, like the stellar temperature $T_{\star}$, stellar luminosity $L_{\star}$ and stellar mass $M_{\star}$, are additional model parameters but these quantities are well constrained by previous studies (see Table 1).

\subsection{Active disk}

Our active disk model is a passively heated (dust) disk where accretion effects are added. The existing MC3D radiative transfer code therefore has to be extended. The implemented accretion effects are briefly described in Appendix A.

Apart from the parameters of the disk and the star, our accretion model requires three additional model parameters: accretion rate $\dot{M}$, boundary temperature $T_{\text {bnd }}$ of the accreting regions on the surface of the star, and a magnetic truncation radius $R_{\text {bnd }}$. Defining the inner radius of the gaseous disk inside $R_{\text {in }}$,

\footnotetext{
${ }^{6}$ We assume a gas-to-dust mass ratio of 100:1.
}

Königl (1991) showed that the truncation radius is not an independent quantity, but depends on stellar radius, mass, accretion rate and magnetic field strength. However, as we do not know the exact magnetic field strength of RY Tau, we fix the boundary temperature and truncation radius to $8000 \mathrm{~K}$ and $5 R_{\star}$, respectively. Both values were already used for the same object by the study of Akeson et al. (2005) and were justified by the assumed large magnetic field of RY Tau in the range of a few kilo-Gauss.

The best fit parameters for this model are given in Table 4 and the model is compared to the observations in Fig. 6. The accretion luminosity is $1.2 L_{\odot}$. This model suffers from the following deficiency: the far-infrared (FIR) wavelength range in the SED is slightly overestimated. A potential improvement of this active disk model could be a "truncated outer disk", formerly suggested by Lucas \& Roche (1997) and recently used by Preibisch et al. (2006). For this, the primary density distribution of the disk is truncated at an outer radius $R_{\mathrm{TD}}$ by multiplying the surface density $\Sigma(r)$ (Eq. (2)) with a Fermi-type function. With a constant $C_{\mathrm{TD}}>0$

$\Sigma_{\mathrm{TD}}(r)=\frac{\Sigma(r)}{1+\exp \left(C_{\mathrm{TD}} \frac{r}{R_{\mathrm{TD}}}-C_{\mathrm{TD}}\right)}$.

This decreases the extended IR emission while the inner disk structure is modified much less than the outer disk regions. It could have its physical origin in a stellar companion which truncates the outer disk region. In fact, Bertout et al. (1999) pointed to a potential binarity of the system. Our study has shown that only a truncation radius of the order of 10 AU could effectively decrease the computed SED (Preibisch et al. 2006). However, this truncation radius is still too large to correspond to the findings of Bertout et al. (1999). Moreover, such a truncation radius is so small that the MIR visibilities increase. Another possibility to decrease the extended IR emission is dust settling that results in a flattening of the flared disk as suggested by Miyake \& Nakagawa (1995) and Dullemond \& Dominik (2006). As outer disk regions are less heated, a less effective flared disk would also decrease the visibility.

Studying SED and visibility, the inclination $\vartheta$ and the position angle PA of the object cannot be clearly derived. Two visibility points are not sufficient to derive these values. With respect to the SED only an upper limit of $\leqq 65^{\circ}$ can be determined. This corresponds to the angle of the line of sight where the optical depth $\tau_{\mathrm{V}}$ in the model exceeds unity.

The outer disk radius $R_{\text {out }}=270 \mathrm{AU}$ of this model is larger than the results from previous measurements. 


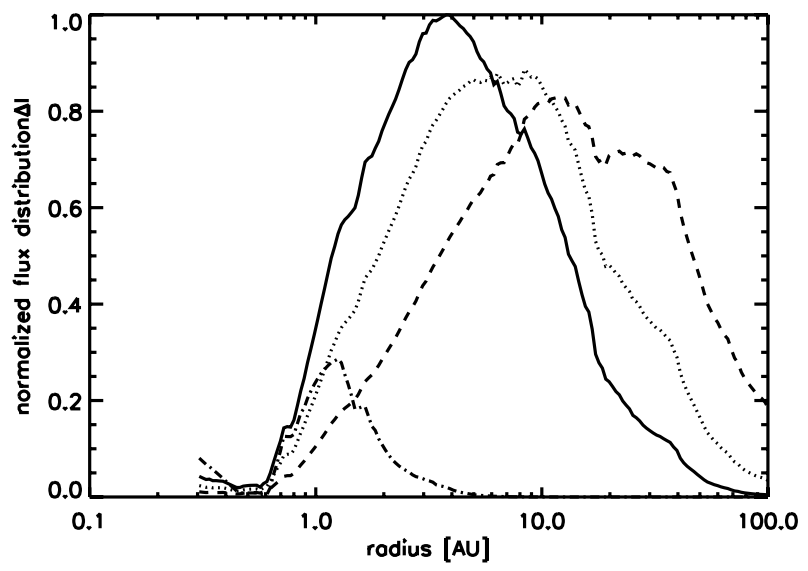

Fig. 2. Radial flux distribution $\Delta I / \Delta r$ for a wavelength of $8 \mu \mathrm{m}$ (solid), $10 \mu \mathrm{m}$ (dotted), $12 \mu \mathrm{m}$ (dashed curve) and $2.4 \mu \mathrm{m}$ (dashed-dotted curve). The mean flux $I$, emitted at a radius $r$, is multiplied with $4 \pi r^{2}$ and normalized by the maximum of the radial flux distribution $I$ at a wavelength of $\lambda=8 \mu \mathrm{m}$.

Andrews \& Williams (2006) derived an outer disk radius of $150 \mathrm{AU}$ with the Submillimeter Array (SMA), while Rodmann et al. (2006) found an outer disk radius of only $(90 \pm 15)$ AU with 7-mm continuum observations at the Very Large Array (VLA). Figure 2 shows at which stellar distances the MIR flux arises in our model. This radial flux distribution confirms that it has its origin in the inner disk regions $(<10 \mathrm{AU})$, mainly, that can only be observed with interferometric methods. But it also shows that MIDI is not sensitive to outer disk regions ( $>40 \mathrm{AU}$ ), including the outer disk radius. A modeling of mm maps of this object allows to determine the outer disk radius but this is out of the scope of this paper. Additionally, it should be underlined that the distribution of the NIR flux from the disk culminates at $\sim 1 \mathrm{AU}$ for this model.

The disk mass of this model $M_{\text {disk }}=4 \times 10^{-3} M_{\odot}$ is by a factor of 3 smaller than the value that was found by Akeson et al. (2005). In this context we have to underline that the disk mass strongly depends on the dust set that is used in the model (see Sect. 4.2). The Fig. 3, left, shows the SED for our active disk model but with the MRN dust set $\left(a_{\max }=0.25 \mu \mathrm{m}\right)$, only. All other model parameters are adapted. In this figure it can be seen that the resulting flux in the $\mathrm{mm}$ wavelength range strongly declines for $a_{\max }=0.25 \mu \mathrm{m}$.

Finally, we attempted to reproduce SED and $N$ band visibilities with a passive disk model, solely, instead of implementing accretion effects, additionally. Such passive disk models for different parameter sets were calculated but without reproducing the SED and MIR visibilities, sufficiently. Each modeled SED of these simulations suffers from similar deficiencies (see upper panel in Fig. 6): in contrast to the photometric measurements the predicted NIR flux between $\lambda \approx 3 \mu \mathrm{m}$ and $8 \mu \mathrm{m}$ is generally underestimated. Moreover, the model is too strongly spatially resolved (visibilities which are too low) in comparison with the measured visibilities.

This lack of NIR radiation in "naked" passive disk models was reported by Hartmann et al. in 1993. Obviously, accretion can generate the missing NIR excess in a region which cannot resolved by the interferometer. In the following subsections we present two further modifications (the puffed-up inner rim wall, an envelope) which have been considered to reproduce distinct NIR excess.

\subsection{Inner rim wall}

In addition to an accretion model that is described in Sect. 5.1, Natta et al. (2001) suggested that a puffed-up wall at the inner disk rim could also produce an increased NIR excess. Dust at the inner edge of the disk is strongly heated by direct stellar irradiation. Based on the idea of hydrostatical equilibrium this heating of the inner disk edge is assumed to cause an expansion of the dust layers perpendicular to the midplane of the disk. Dullemond et al. (2001) established an analytical model of the puffed-up, inner rim wall as a supplement to the Chiang-Goldreich model (Chiang \& Goldreich 1997) and defined the "inner rim scaleheight" $H_{\text {rim }}$ as follows:

$H_{\text {rim }}=\chi_{\text {rim }} h_{\text {in }}$,

where $\chi_{\text {rim }}$ is a dimensionless factor, larger than unity. The quantity $h_{\text {in }}$ represents the scale-height of the inner disk without extra vertical expansion ${ }^{7}$. Simulations of young stellar objects (YSOs) where the inner rim wall has been successfully applied, have been published by Dominik et al. (2003), Pontoppidan et al. (2005), and Eisner et al. (2005a), for instance. The reproduction of interferometric observations in the NIR regime assuming simple, analytical ring models (Millan-Gabet et al. 2001) has also been used as a further confirmation of the inner rim wall (Natta et al. 2001). Furthermore, a puffed-up inner rim, shadows adjacent areas of the disk from direct stellar radiation ("selfshadowed disk"). This effect is used to explain the spectral shape of the FIR excess from YSOs and to explain the classification of Group I and Group II sources (Meeus et al. 2001; Dullemond \& Dominik 2004a). Arising from a flared disk a Group I source has a flat SED over the entire infrared wavelength range. The SED of a Group II source, however, strongly declines towards the FIR. Such a decline was explained by a self-shadowed disk.

The puffed-up inner rim wall is still a controversial topic (e.g., Millan-Gabet et al. 2006). In particular, it was shown that a puffed-up inner rim wall does not generally emit enough radiation to cause the observed NIR excess in contrast to an envelope similar to the one that we implement in Sect. 5.3 (Vinković et al. 2006). Another open issue is the static stability of the proposed sharp, inner rim wall (Dullemond et al. 2001). Considering the sublimation temperature of the used dust species, Isella \& Natta (2005) revised the previous inner rim model by a more rounded-off inner rim. Monnier et al. (2006) observed RY Tau in the NIR wavelength range using the Infrared Optical Telescope Array (IOTA) where a spatial resolution of $\geqq 1$ AU has been reached, comparable to our MIDI observations. In fact, their modeling results were incompatible with the models possessing vertical inner walls.

In our model the vertical density distribution is calculated assuming hydrostatic equilibrium. Therefore, the potential formation of a puffed-up inner wall is included in a natural way. However, it requires special computational care. In order to detect the effect of the puffed-up inner rim wall the size of grid cells in the inner region of the disk model should be small enough. A too coarsely meshed grid results in too low, averaged cell temperatures and in the absence of a potential puffedup inner rim wall. We use a polar coordinate system $(r, \theta)$ in our two-dimensional model with uniform steps in $\theta\left(\Delta \theta=1^{\circ}\right)$ and a logarithmic scale for $r$ (most inner step $\Delta r \approx 0.01 \mathrm{AU}$ ). Therefore, the inner grid cells have an approximate size of

\footnotetext{
7 The scale-height is defined as the vertical distance from the midplane where the density has decreased by a factor $e \approx 2.718$ (Euler's constant).
} 

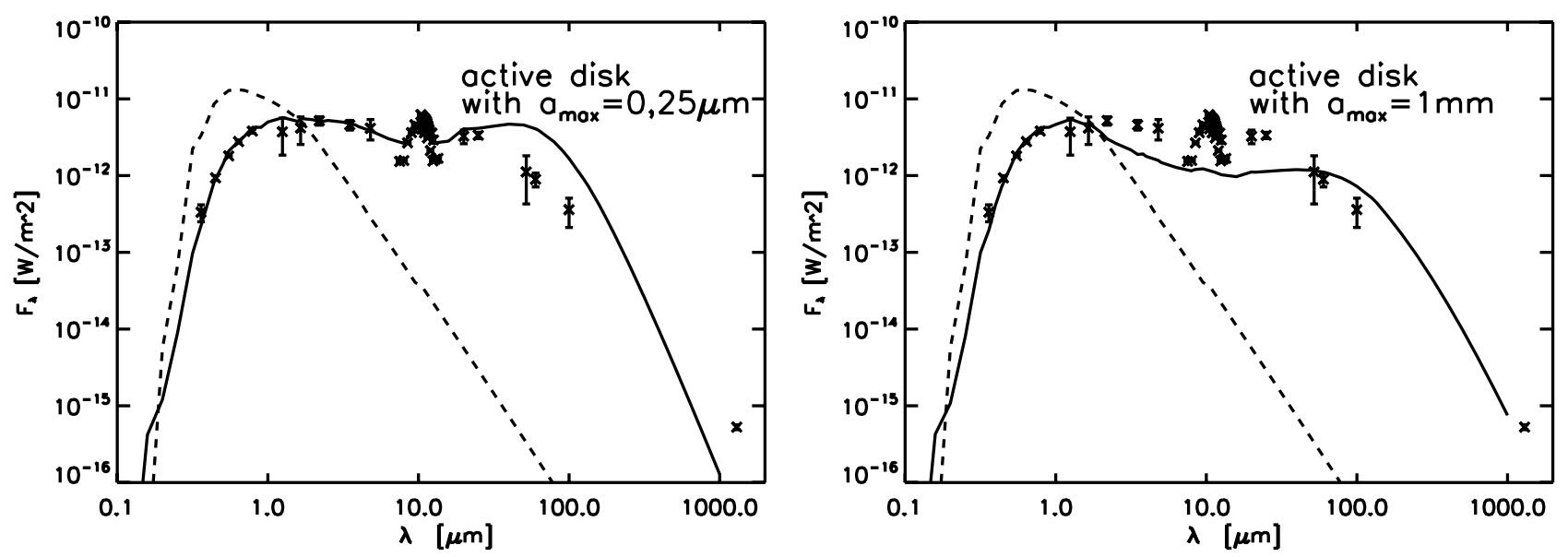

Fig. 3. Left: deficiency of the active disk model at long wavelength if only dust with standard size MRN distribution is assumed $\left(a_{\max }=0.25 \mu \mathrm{m}\right.$; compare middle panel in Fig. 6). Observations are represented by crosses with error bars (s. Table 2). The predicted slope into the mm range is too steep. The dashed line shows the unreddened stellar flux. Right: the same as the left figure but an maximum dust grain radius of $a_{\max }=0.25 \mu \mathrm{m}$ is assumed.

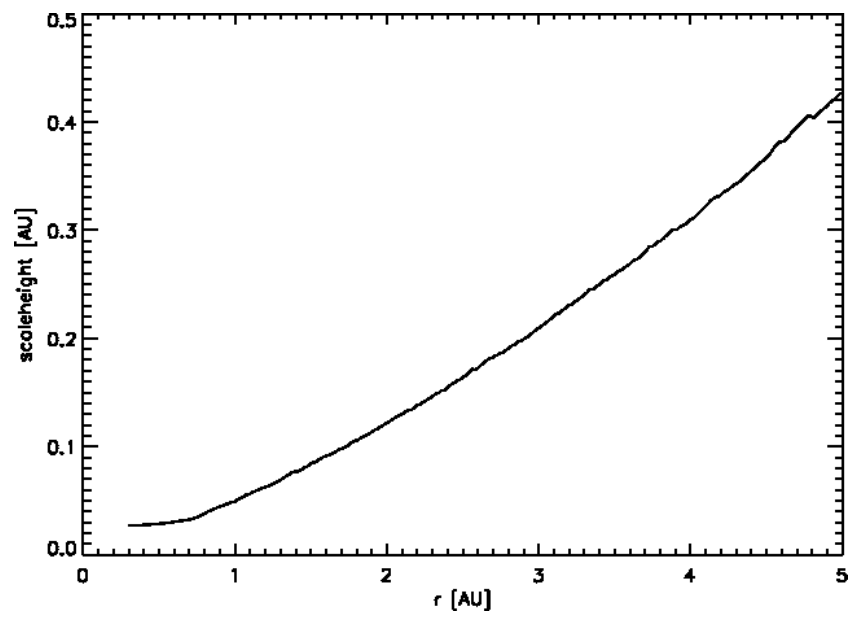

Fig. 4. Scaleheight versus disk radius of our disk model from Sect. 5.1. We do not see a puffed-up inner rim in the sense of a local maximum of the scaleheight at the inner rim and a following local minimum at slightly larger radii although the rim of our model catches a large fraction of stellar radiation.

$\sim(0.01 \times 0.005) \mathrm{AU}^{2}$. According to Dullemond et al. $(2001)$ a puffed-up scaleheight of $0.05 \mathrm{AU}$ up to $0.10 \mathrm{AU}$ can be expected. Figure 4 shows the scale-height of our active disk model of Sect. 5.1.

The fact that we do not see an excessively puffed-up inner wall in our approach does not necessarily exclude such a phenomenon. It is possible that the T Tauri star RY Tau is still too faint. In their study Dullemond \& Dominik (2004a) assumed HAeBe stars with temperatures in the range of $T_{\text {eff }}=10000 \mathrm{~K}$ and luminosities up to several 10 solar luminosities (see also Dominik et al. 2003). The optical depth of the inner disk region also affects the formation of a rim wall. If the optical depth is small at the inner disk region, the radiation is not absorbed mainly at the inner rim of the disk but on a larger scale. In this context Dullemond \& Dominik (2004a) showed that the vertical height of the inner rim wall is notably boosted for exponents $p \approx 4$ for the surface density (Eq. (2)). Finally, the properties of the dust grains that are used in the modeling approach can also affect the inner rim as Vinković et al. (2006) mentioned. In Fig. 2 it is shown that the inner rim of our model emits only a smaller fraction of the NIR radiation.

Our simulations are based on a Monte Carlo approach. Its drawback is the presence of statistical noise in the result which could in principle blur an effect such as the puffed-up inner rim wall. In order to quantify an upper limit of the scaleheight of a potential puffed-up inner rim, we assume that the scaleheight at the innermost 0.3-3 AU can be described by a quadratic polynomial $\left(h \propto r^{2}\right)$. This polynomial is then fitted to the scaleheight. The corresponding standard deviation $\sigma_{\mathrm{SD}}$ between the quadratic function and the scaleheight represents the maximum height of a potential puffed-up inner rim. We get $\sigma_{\mathrm{SD}} \leqq 0.002 \mathrm{AU}$ which is much smaller than the value derived by Dullemond et al. (2001).

The strength of the inner rim wall is still an open issue. It depends strongly on dust properties, radial disk structure and stellar properties that are used in the models. Future, highly spatially resolved observations in the NIR wavelength regime, that are sensitive to the hot inner edge of the disk, will us allow to decide to what extent the effect of a puffed-up inner disk wall exists and which observational effects are provoked by this phenomenon.

\subsection{Active disk with a dusty envelope}

Many studies (e.g., Hartmann et al. 1993; Calvet et al. 1997) have shown that a dusty envelope around YSOs and Class-I sources, in particular, substantially contributes to the observed NIR excess. With respect to its optical depth a dusty envelope could even dim the stellar radiation in the visible wavelength regime.

There are also several studies which justify an envelope structure around the star and disk of RY Tau. Vink et al. (2003) found that changes of the polarization across the $\mathrm{H} \alpha$ line of RY Tau are based on scattering effects due to an extended dusty envelope. Direct evidence for such a circumstellar halo has been provided by $R$ and $I$ coronagraphic, large-scale images of RY Tau (Nakajima \& Golimowski 1995), in particular, and NIR, scattered light images around different YSOs (e.g., Padgett et al. 1999; Allen et al. 2002), in general. Certainly, one challenge for interferometric studies is to decide whether the observed large-scale halo around RY Tau extends down to the inner disk region. 
In the context of axisymmetric accretion models Ulrich (1976) created an infall model of circumstellar gas and dust in an envelope structure in order to reproduce the emission-line $\mathrm{H} \alpha$ and $\mathrm{H} \beta$ profiles of type I/II P Cygni objects. This ansatz has been successfully used in modeling infrared images of Class-I objects (e.g., Lucas \& Roche 1997; Wolf et al. 2003). In contrast to Ulrich's approach we add a more simple spherical dust configuration to the disk model. The spherical envelope in our model is geometrically constrained by the inner $\left(R_{\text {in }}\right)$ and outer $\left(R_{\text {out }}\right)$ disk radius. With the density distributions of the disk $\rho_{\mathrm{disk}}(r, \theta)$, of the envelope $\rho_{\mathrm{env}}(r, \theta)$, and the the position vector $\boldsymbol{r}$ as well as the coordinates $r$ and $\theta$ we define

$\rho_{\text {env }}(r, 0)=c_{1} \cdot \rho_{\text {disk }}\left(R_{\text {in }}, 0\right) \cdot\left(\frac{|\boldsymbol{r}|}{R_{\text {in }}}\right)^{-c_{2}}$

where $c_{1} \ll 1$ and $c_{2}>0$. The constraint $c_{1} \ll 1$ guarantees a low optical depth of the envelope and the possibility to observe the innermost region of the disk. Disk and envelope are combined by

$$
\begin{array}{lll}
\rho(\boldsymbol{r})=\rho_{\mathrm{disk}}(\boldsymbol{r}) & \text { for } & \rho_{\mathrm{env}}(\boldsymbol{r}) \leqq \rho_{\text {disk }}(\boldsymbol{r}) \\
\text { and } & & \\
\rho(\boldsymbol{r})=\rho_{\mathrm{env}}(\boldsymbol{r}) & \text { for } & \rho_{\mathrm{env}}(\boldsymbol{r})>\rho_{\text {disk }}(\boldsymbol{r}) .
\end{array}
$$

This assumption ensures a smooth transition from disk to envelope. In this simple envelope + active disk model we do not implement bipolar cavities although there are hints that such cavities, which are caused by collimated outflows, i.e. jets, generally exist in YSOs (Edwards et al. 1993). Actually, the number of free modeling parameters should be as small as possible to ensure that we do not overdetermine our approach. Furthermore, based on images of YSOs at a similar evolutionary state as RY Tau, Eisner et al. (2005b) mentioned that cavities have effects on the structure of scattered light emission, which has its maximum in the NIR wavelength range.

Figure 6 and Table 4 show the result and parameter set of our best envelope + active disk models. The accretion rate of the model $\dot{M}=2.5 \times 10^{-8} M_{\odot} \mathrm{yr}^{-1}$ is by a factor of $\sim 4$ smaller than the accretion rate assumed in the previous model without envelope. However, both models reproduce the SED and the visibilities. This result shows that both accretion and an envelope, have the same effects on the SED and visibilities in the NIR and MIR wavelength range. Moreover, we have to mention that the measurements could be reproduced without considering any accretion effects. A comparison of these results follows in Sect. 7. The accretion luminosity is $0.3 L_{\odot}$. In the visual range and for inclinations $\vartheta \leqq 65^{\circ}$ the model is optically thin as can be seen in Fig. 5. However the envelope evokes an observational effect on the SED and MIR-visibilities.

\section{Radial gradients of the dust composition in circumstellar disks}

As mentioned in Schegerer et al. (2006) a more advanced disk model considers the specific dust composition of the corresponding object instead of using a canonical MRN dust set with averaged optical quantities (see Sect. 4.2). In such dust models the following dust components are generally taken into account: carbon, which mainly contributes to the underlying continuum as well as amorphous and crystalline silicate dust which generates the silicate features at $\sim 10 \mu \mathrm{m}$ and $\sim 20 \mu \mathrm{m}$. Other dust species such as water ice or, more precisely, water ice mantles

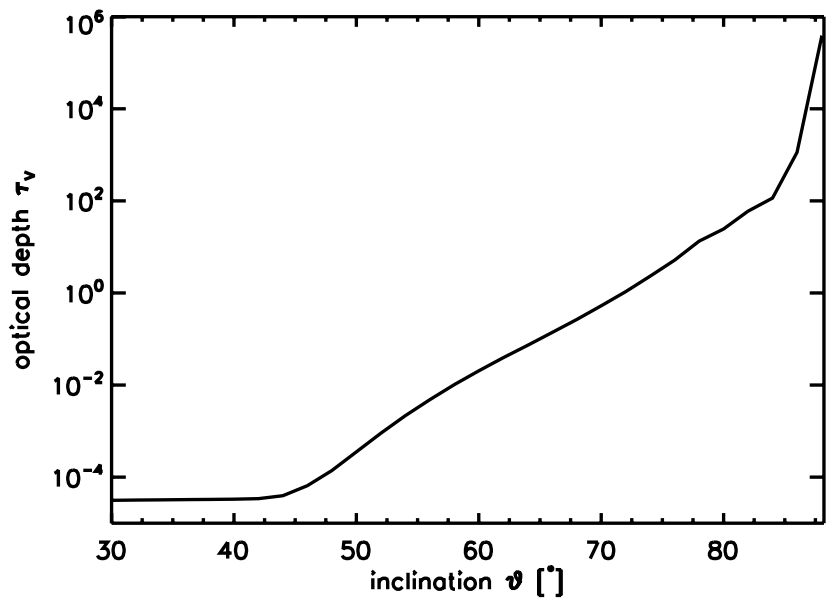

Fig. 5. Optical depth for a inclination of $\vartheta$ in the optical wavelength range. For smaller inclinations the optical depth is smaller than unity ensuring the observations of the inner regions. However, this envelope still has effects on the SED and MIR visibilities.

around carbon/silicate grains can also be considered (see Fig. 1 in Chiang et al. 2001).

First comparisons between the silicate feature measured in laboratory experiments and observationally-based silicate spectra of YSOs have been drawn by Jäger et al. (1994). A widely accepted analysis to determine the silicate composition of circumstellar dust is a $\chi^{2}$-fitting method that was established by Bouwman et al. (2001). They assumed that the silicate emission feature has its origin in the optically thin surface layer of the circumstellar disk where it results from a linear combination of mass absorption coefficient (emissivity) $\kappa_{i}$ of different dust components $i$ :

$F(v)=B(v, T)\left(C_{0}+\sum_{i=1}^{n} C_{i} \kappa_{i}(v)\right)$,

where $C_{0}$ and $C_{i}$ are fitting parameters which reflect the mass contribution of each component $i$. The quantity $F(v)$ is the spectral flux at frequency $v, \kappa_{i}(v)$ represents the frequency-dependent mass absorption coefficient for dust component $i$ and $B_{v}(T)$ is the Planck function corresponding to a blackbody temperature $T$. As basic dust set for their $\chi^{2}$-fitting routine for T Tauri objects Schegerer et al. (2006) used the following silicate species: small $(0.1 \mu \mathrm{m})$ and large $(1.5 \mu \mathrm{m})$ grains of amorphous olivine, and pyroxene, as well as crystalline species such as forsterite, enstatite, and quartz.

The single-dish spectrum $F_{v}$ is measured with a single telescope. For each interferometric observation of RY Tau we obtained a correlated spectrum (s. Eq. (1)) which reflects the flux emitted by a region which was not spatially resolved by the interferometer. An increasing effective baseline length of the interferometer results in a higher resolution. It has to be pointed out, that the single-dish spectra as well as the correlated spectra contain spectral contributions of the silicate emission from the whole disk, but the contributions from the hotter and brighter regions are increasing with increasing effective baseline length. In this context a homogeneous, axial-symmetric disk is assumed.

According to the method used in Schegerer et al. (2006), we find a decreasing contribution of not-evolved, i.e. amorphous, $0.1 \mu \mathrm{m}-$ small dust grains and an increasing crystallinity with increasing baseline length, i.e. decreasing distance to the central T Tauri star (see Fig. 7, Table 5). For comparison we add 

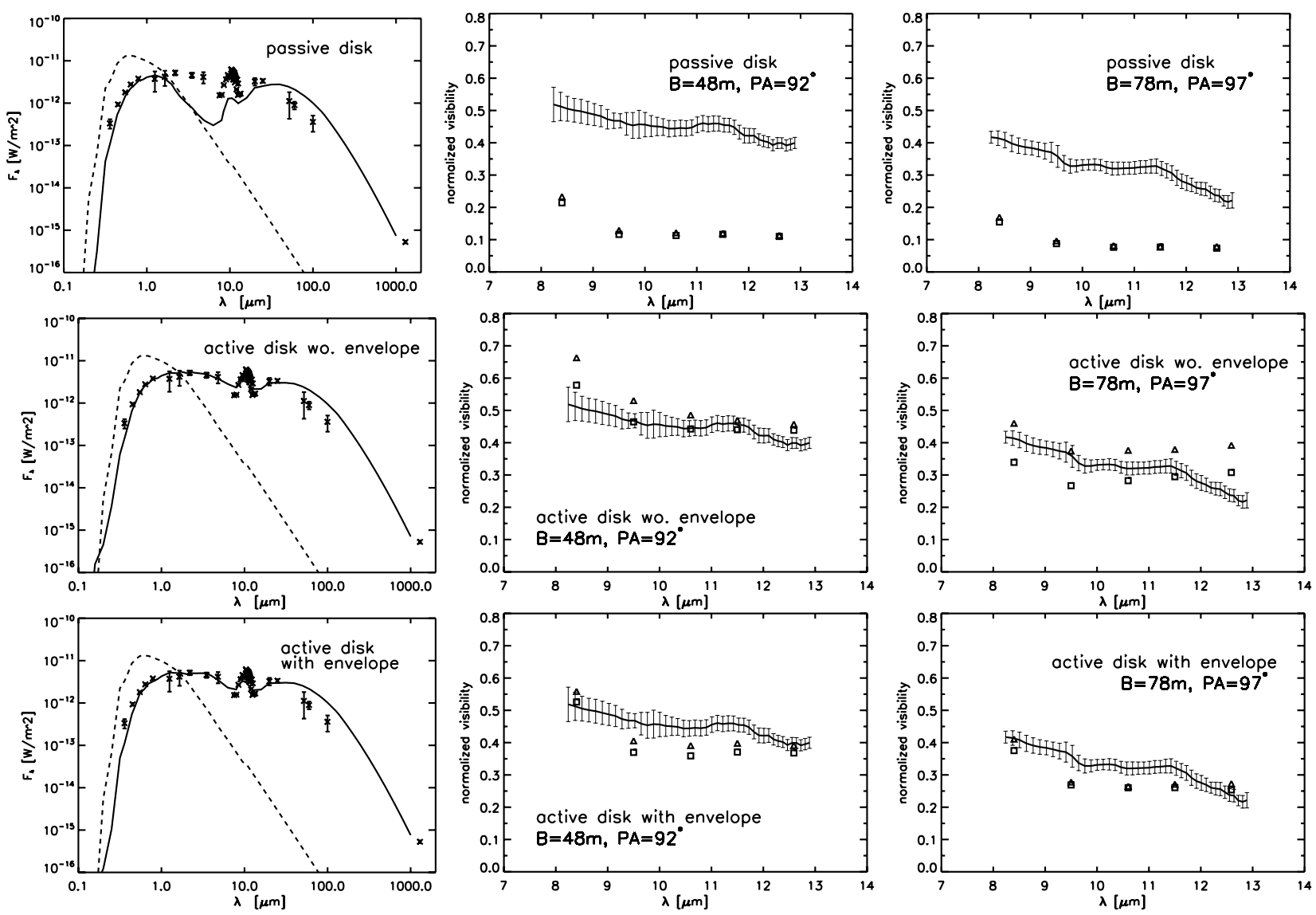

Fig. 6. Top row: models of the spectral density distribution of RY Tau for an inclination angle of $\vartheta=25^{\circ}$. Here, we assume a "naked", passively heated disk model without considering accretion effects and an envelope. Real photometric data with error bars are included in both models (see Table 2). The dashed line represents the intrinsic, stellar flux. The flux $F_{\lambda}$ is given in units $\mathrm{W} \mathrm{m}^{-2}$. According to the theorem of van CittertZernicke the modeled visibilities in all diagrams were calculated from the corresponding model image for the wavelengths of 8.5, 9.5, 10.6, 11.5 and $12.5 \mu \mathrm{m}$. Triangles and squares represent the upper and lower limit of the visibilities $V(\lambda)$ for different position angles but the same inclination of the model. The measured data are included with error bars. Middle row: model of the spectral density distribution of RY Tau for an inclination of $25^{\circ}$ considering accretion effects in addition to a passively heated disk. Bottom row: SED that is obtained only from an active disk model considering an envelope, additionally.

the single-dish, i.e. non-correlated spectrum $F_{v}$ in Fig. 7. This single-dish spectrum confirms the derived tendencies.

Figure 8 shows the crystallinity $C_{\text {crys }}$, which is plotted versus the spatial resolution of our MIDI observations. The crystalized material is concentrated mainly in the inner parts of the disk (point $\mathrm{C}$ for highest resolution), decreases strongly with decreasing resolution (point $\mathrm{B}$ for the intermediate resolution) and converge to a lower limit for the single-dish observation (point A) which corresponds approximately to the abundance of crystalline dust in interstellar matter (Gail 2003). The relative mass contribution of small dust grains decreases from the outer to the inner disk regions. Considering the used spectroscopic slit we assume a resolution of $0.52^{\prime \prime}$ for the single-dish observations. A corresponding result was previously found by van Boekel et al. (2004) for several HAeBe stars indicating more evolved silicate dust towards inner disk regions. Our result shows that the formation of crystalized dust grains is also favored in the innermost disk region of T Tauri stars. A study of the absolute disk position of the crystalized dust, as for any other material, is out of the scope of this paper and should be presented in a future publication. Nonetheless such a forthcoming study is favored by the fact that the position angles of our observations with MIDI are almost identical (Table 3), i.e. $C_{\text {crys }}$ depends only on the radial coordinate $r$ in the disk. Such a study
Table 5. Results of our $\chi^{2}$-fit presented in Fig. 7. The used method is described in Schegerer et al. (2006) in detail. The underlying continuum is estimated by a single blackbody function with the temperature $T$. "RMC" stands for relative mass contribution, "am.-sma." for amorphous, $0.1 \mu \mathrm{m}$-small, "am.-la." for amorphous, $1.5 \mu \mathrm{m}$-large and "crys." for crystalline silicate dust grains. The crystalline component includes small and large silicate species: forsterite, enstatite, and quartz. See text for further discussion.

\begin{tabular}{|c|c|c|c|c|}
\hline & Baseline length & Resolution & Silicate & RMC \\
\hline A & single-dish & $\begin{array}{c}70 \mathrm{AU} \\
(520 \mathrm{mas})\end{array}$ & $\begin{array}{c}\text { am.-sma. } \\
\text { am.-la. } \\
\text { crys. } \\
T\end{array}$ & $\begin{array}{r}(15 \pm 3) \% \\
(82 \pm 3) \% \\
(3 \pm 1) \% \\
(450 \pm 3) \mathrm{K}\end{array}$ \\
\hline B & $49 \mathrm{~m}$ & $\begin{array}{c}2.8 \mathrm{AU} \\
(21 \mathrm{mas})\end{array}$ & $\begin{array}{c}\text { am.-sma } \\
\text { am.-la. } \\
\text { crys. } \\
T\end{array}$ & $\begin{array}{r}(7 \pm 3) \% \\
(82 \pm 5) \% \\
(11 \pm 2) \% \\
(557 \pm 5) \mathrm{K}\end{array}$ \\
\hline $\mathrm{C}$ & $78 \mathrm{~m}$ & $\begin{array}{c}1.8 \mathrm{AU} \\
(13 \mathrm{mas})\end{array}$ & $\begin{array}{c}\text { am.-sma } \\
\text { am.-la. } \\
\text { crys. } \\
T \\
\end{array}$ & $\begin{array}{r}(1 \pm 1) \% \\
(80 \pm 4) \% \\
(19 \pm 2) \% \\
(722 \pm 9) \mathrm{K}\end{array}$ \\
\hline
\end{tabular}

would be an essential requirement to study the degree of radial mixing of material in circumstellar disks described by the ratio 

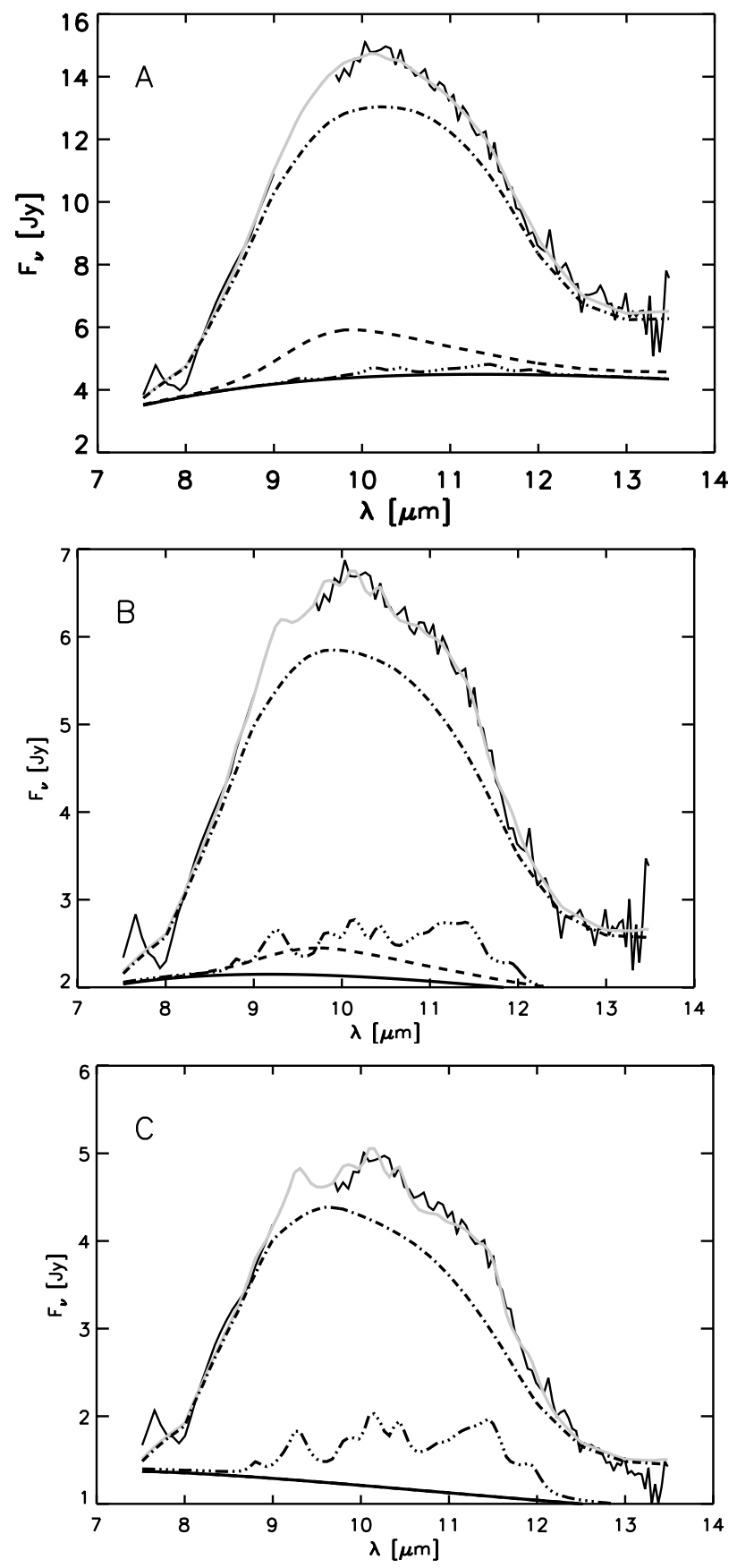

Fig. 7. Single-dish (A) and correlated spectra $(\mathbf{B})$ and $(\mathbf{C})$ of the T Tauri star RY Tau (black, solid lines). The correlated spectra (B) and (C) corresponds to a baseline length of $49 \mathrm{~m}$ and $78 \mathrm{~m}$, respectively. The silicate feature are modeled by a linear combination of mass absorption coefficients $\kappa_{i}$ of different amorphous and crystalline silicates (grey lines). In order to exclude remnants of the data reduction we cut the wavelength interval $\sim 9.0 \mu \mathrm{m}$ to $\sim 9.7 \mu \mathrm{m}$ influenced by the telluric ozone band. Dashed lines and dot-dashed lines represent the contribution of $0.1 \mu \mathrm{m}$ - and $1.5 \mu \mathrm{m}$-sized, amorphous grains, respectively. The dot-dotdashed curves stand for the crystalline contribution. The underlying, solid curves represent the continuum (here: blackbody with temperature $T$ ).

of the viscous inward and diffusive outwards stream (Wehrstedt \& Gail 2002; Bouwman et al. 2003; Gail 2004; Pavlyuchenko $\&$ Dullemond 2007). Moreover, such a study could give a hint whether circumstellar dust grains are only crystalized by thermal heating in the inner disk regions or whether electric discharges in

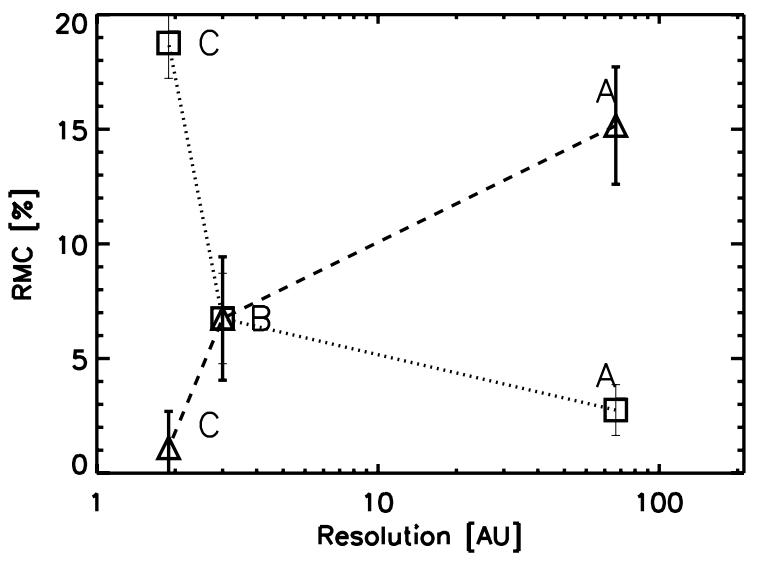

Fig. 8. RMC of crystalized (dotted line, squares) and $0,1 \mu \mathrm{m}$-small, amorphous (dashed line, triangles) silicate grains plotted versus the reached spatial resolution of our observations.

outer disk regions can also crystalize dust grains. Both, a strong radial diffusion and electric discharges in outer disk regions, respectively, would imply a shallow decrease of the crystallinity with radius. Although Fig. 8 qualitatively shows that crystalized dust grains are located mainly in the inner disk region, a further study is required to determine the absolute disk position of the crystallised dust, along with more interferometric observations with different spatial resolutions.

Finally, we add a few comments on the dust set used in our modeling approach. Instead of using the canonical dust set of "astronomical silicate" and carbon, we could also use the specific silicate dust composition found by the simple linear fitting routine presented above. Preibisch et al. (2006) have already used such an approach for modeling the HAeBe star HR 5999. However, this proceeding is problematic in light of following arguments:

i. The best-fit model parameters are not sensitive to very small grains $(<0.1 \mu \mathrm{m})$. But such tiny grains are essential for generating the observed NIR emission as Weingartner \& Draine (2001) pointed out. As mentioned in Sect. 4.2, a dust set where large dust particles $a>1 \mu \mathrm{m}$ are implemented at the expense of tiny dust grains, is very ineffective in generating NIR and MIR flux. Only additional modifications of the inner disk structure can compensate this lack of emission. However, in disk systems where the NIR flux has already vanished, the substition of small grains by large grains could be physically justified by advanced dust coagulation (Weinberger et al. 1999; Kornet et al. 2001).

ii. The best-fit model parameters are not sensitive to carbon. The emission profile of carbon is strictly monotonic in the $10 \mu \mathrm{m}$-wavelength range (e.g., Wolf \& Hillenbrand 2003) and thus contributes to the underlying continuum, only. If carbon is considered in Eq. (7), too, each potential contribution $C_{\text {carbon }}$ of carbon would not be independent of the contribution of the single blackbody function used to reproduce the underlying continuum.

iii. Apart from the analysis of the contributions of each silicate component, another important result of our $10 \mu \mathrm{m}$-analysis is the derived tendency of increasing crystallinity towards inner disk regions. However, this result does not enclose the determination of the absolute disk position of the crystallised dust and it would be another requirement to use the results of our $\chi^{2}$-fitting routine in our modeling approach. 
iv. It has not been clear, so far, if the determined contribution of each dust component corresponds to its mass fraction. As shown in Schegerer et al. (2006) the porosity of dust grains, which is not considered in our $\chi^{2}$-fit, affects the shape of the silicate feature similar to the size effect. Therefore, the mass contributions of large, compact dust grains could be overestimated while the increasing crystallinity of the dust in the inner regions is still a safe conclusion (Sect. 6).

Only further studies can clarify if and how the results of the presented $\chi^{2}$-fitting routine can be implemented in the modeling approach.

\section{Discussion}

The main aim of this paper was to model the structure of the circumstellar environment of the T Tauri star RY Tau. In this context we presented different modeling approaches for the circumstellar dust distribution (passive disk; active disk; active disk + envelope) and pointed to potential supplements such as the puffed-up inner rim wall or the truncated outer disk model. An important aim of our approach was to keep the number of model parameters as small as possible. With respect to our modeling results additional parameters and modifications were only implemented if significant improvements could be obtained afterwards. In order to decrease the mm slope of the resulting SED, the disk with the MRN dust composition of astronomical silicate and carbon were replaced by a two-layer dust model where the disk interior also contains evolved, i.e. larger dust grains.

\subsection{The merits of the active disk model with and without an envelope}

Accretion or dusty envelopes produce the additional infrared flux from $\lambda \approx 2 \mu \mathrm{m}$ up to $\lambda \approx 8 \mu \mathrm{m}$ of the SED that is missed in the model of a "naked", passively heated disk.

The extra infrared radiation from the active disk model is generated in the innermost disk region inwards the point of dust sublimation (Appendix A). The radiation which is caused by accretion additionally heats the innermost disk regions close to the inner disk radius. In comparison to a "naked", passively heated disk, the implementation of accretion effects in the model results in a higher spatial concentration of infrared flux in the inner region that is not spatially resolved by our MIDI measurement. The computed MIR visibility increases, therefore (compare middle and lower panel in Fig. 6). The accretion rate of the model presented here (active disk without envelope), $9.1 \times 10^{-8} M_{\odot} \mathrm{yr}^{-1}$, is smaller than the value found by Akeson et al. $\left(2005 ; 2.5 \times 10^{-7} M_{\odot} \mathrm{yr}^{-1}\right)$ in their model study but corresponds to that of Calvet et al. $\left(2004 ; 6.4-9.1 \times 10^{-8} M_{\odot} \mathrm{yr}^{-1}\right)$. The latter result is based on a multi-wavelength study in the optical-UV range considering different emission-line profiles. An accretion rate of $2.5 \times 10^{-7} M_{\odot} \mathrm{yr}^{-1}$ decreases the visibilities in the MIR range because of a stronger irradiation of outer disk regions. Based on a flux ratio measurement between the continuum excess and the intrinsic photospheric flux at a wavelength of $5700 \AA$, Hartigan et al. (1995) found a much lower accretion rate of $\dot{M} \approx 2.5 \times 10^{-8} M_{\odot} \mathrm{yr}^{-1}$ in RY Tau. Vink et al. (2003) determined an accretion rate of $\dot{M} \approx 7.5 \times 10^{-8} M_{\odot} \mathrm{yr}^{-1}$. The derived accretion rate from the model of Akeson et al. (2005) is up to a factor of 10 larger than the values that were measured in the UV range. Such a discrepancy between standard accretion disk models and the measurements has been already discussed by Muzerolle et al. (2004). To obtain a consistency in the measurements, they introduced an artificially puffed-up inner rim in their modeling approach, accounting for the large NIR excesses of classical T Tauri stars, but without requiring excessive accretion rates. Instead of implementing such a puffed-up inner rim we assumed an envelope in our active disk + envelope where we assumed a smaller accretion rate (factor $\sim 4$ ) than in the pure active disk model.

NIR flux in the model with the envelope has its origin close to but outwards of the sublimation point. This extra flux results from the stellar heating of the dust in the envelope up to the sublimation temperature and in an increase of the MIR visibility corresponding to the pure active model.

In contrast to pure accretion, a dusty envelope dims the central star and prevents the outer disk regions from being heated too strongly by direct stellar irradiation. Therefore, the MIR reemission from these outer regions is decreased and the spatial concentration of the infrared radiation in the inner regions is increased. Such an effect caused by the envelope results in an increase of the MIR visibility, in particular for measurements with the smaller projected baseline of $48 \mathrm{~m}$. We have to mention that comparable effects could also be achieved by the truncation of the outer disk in our active disk model or by a strongly puffed-up inner rim.

In contrast to our finding Akeson et al. (2005) did not find any hints for an additional envelope in their modeling approach for RY Tau based on NIR, interferometric observations. They suggest NIR unveiled CO absorption lines which RY Tau is exhibiting. In fact, such unveiled $\mathrm{CO}$ lines could be evidence of the absence of a substantial envelope (Najita et al. 2003; Calvet et al. 1997). However, the almost unveiled CO absorption lines do not necessarily exclude an envelope when considering the following argument:

The results of two-dimensional collapse calculations of the infalling matter in an envelope (Yorke et al. 1993) suggests a more plane-parallel than spherical envelope geometry. In this model, the envelope has already collapsed at the inner disk edge. With MIDI we resolved inner disk regions with a distance of several AU from the star (see Fig. 2) where remnants of the envelope could still exist. However, at these distances the gas in the potential envelope is already too cold to provide a substantial veiling of CO lines. We point to a study of Bastien \& Landstreet (1979) where it was suggested that most of the polarization found towards RY Tau arises from a circumstellar (dusty) envelope which actually lies outside of the hightemperature, gas-emitting region. Therefore, a geometry where the envelope has already disappeared at the inner edge but not at adjacent regions could explain why the observed NIR CO absorption lines are unveiled and why Akeson et al. (2005) failed to model photometric and NIR visibility data considering an additional envelope to their pure active disk model. In fact, with respect to Fig. 2 the NIR emission mainly originates close to the sublimation point.

Another potential origin of such an (not necessarily spherical) envelope could be magnetically driven disk winds containing gas and, additionally, small quantities of small dust particles: material at the disk surface could even follow magnetical field lines for radii $r$ which are much larger than the magnetical truncation radius $r \gg R_{\text {bnd }}$ (Appendix A) and larger than the inner radius $r>R_{\text {in }}$ (Blandford \& Payne 1982). Former studies assumed a correlation between the disk accretion rate $\dot{M}$ onto the star and the outflow mass-loss rate $\dot{M}_{\text {of }}$ with $\dot{M}_{\text {of }} / \dot{M} \sim 0.1$ (Richer et al. 2000). These dust particles that follow the outflowing wind from the disk form the optically thin dusty envelope 
assumed in our modeling approach. A possible consequence of this procedure would be the (acceleration of the) formation of an inner gap in the innermost disk region as observed in older T Tauri objects such as TW Hya (Calvet et al. 2002). Finally, we note that Fendt \& Camenzind (1996) have studied stationary, axisymmetric wind flows driven by a rapidly rotating magnetosphere. They found that the (gas) particle density in the outflow decreases with $r^{-2.3}$. This result is independent from the stellar parameters (private communication with $\mathrm{Ch}$. Fendt).

Another origin of the dust particles in the circumstellar envelope could be hard UV irradiation from the inner accretion zone or/and the star which increases the gas temperature in the upper layers of the disk up to $\sim 10^{4} \mathrm{~K}$ allowing the hot, gaseous material to escape from the gravitationally bound system of the star. Small dust particles accompany the gas outflow. This effect, called photoevaporation, effectively starts at a critical radius $r_{\mathrm{cr}}$ where the sound speed is in the range of the escape speed, i.e. a few AU in the case of RY Tau (see Dullemond et al. 2006, for a review). However, only further theoretical studies can clearify if the mass loss rates of dusty material in these disk outflows is high enough to cause a sufficiently strong effect visible with MIDI. For completeness we note that gas pressure dependent photophoretic forces of light can also induce the ejection of dust from the optical thin surface layers of the disk as studied by Wurm (2005) and Wurm \& Krauss (2006).

YSOs of Class I (Adams et al. 1987) that reveal a circumstellar envelope + disk structure typically also show signs for accretion given that accretion is stronger for younger objects (e.g., Hartmann et al. 2005). Accretion is certainly present in RY Tau, too, according to the mentioned, numerous studies, such as the analysis of the existing $\mathrm{Br} \gamma$ and $\mathrm{H} \alpha$ lines and UV excess radiation. However, we have also noted that the implementation of accretion can be totally ignored in an envelope + passive disk model to reproduce SED and MIR visibilities. Both, accretion and envelope, increase the NIR- and MIR-flux. Only complementary observations in the UV range where the accretion rate can be independently measured, will provide additional constraints to disentangle the different model approaches and allow us to consider both accretion and the envelope in one model.

\subsection{A potential stellar companion?}

As mentioned in Sect. 2, Bertout et al. (1999) found indirect hints for a stellar companion analysing HIPPARCOS data. Assuming the regular motion of the photocenter of RY Tau they derived a projected minimum distance of 3.27 AU and a position angle of $304^{\circ} \pm 34^{\circ}$ for the potential secondary. The method they used is described in Wielen (1996).

A potential detection of a companion by interferometric observations depends on the separation $a_{\text {sep }}$, the position angle of the companion with respect to the position angle of the interferometric baseline and the brightness ratio $a_{\text {rat }}$ of secondary and primary. The visibility of a binary system can be expressed by the approximate formula:

$V(B, \lambda)=a_{0}(B, \lambda) \frac{\sqrt{1+a_{\mathrm{rat}}^{2}+2 a_{\mathrm{rat}} \cos \left(2 \pi a_{\mathrm{sep}} \frac{B}{\lambda}\right)}}{1+a_{\mathrm{rat}}}$.

The parameter $a_{0}(B, \lambda)$ represents the visibility that results from the circumstellar and -binary material assumed to be equally distributed around the components. A positive detection could then be recognized by sinusoidal variation of the visibility (Ratzka 2005). Furthermore, all interferometric observations are
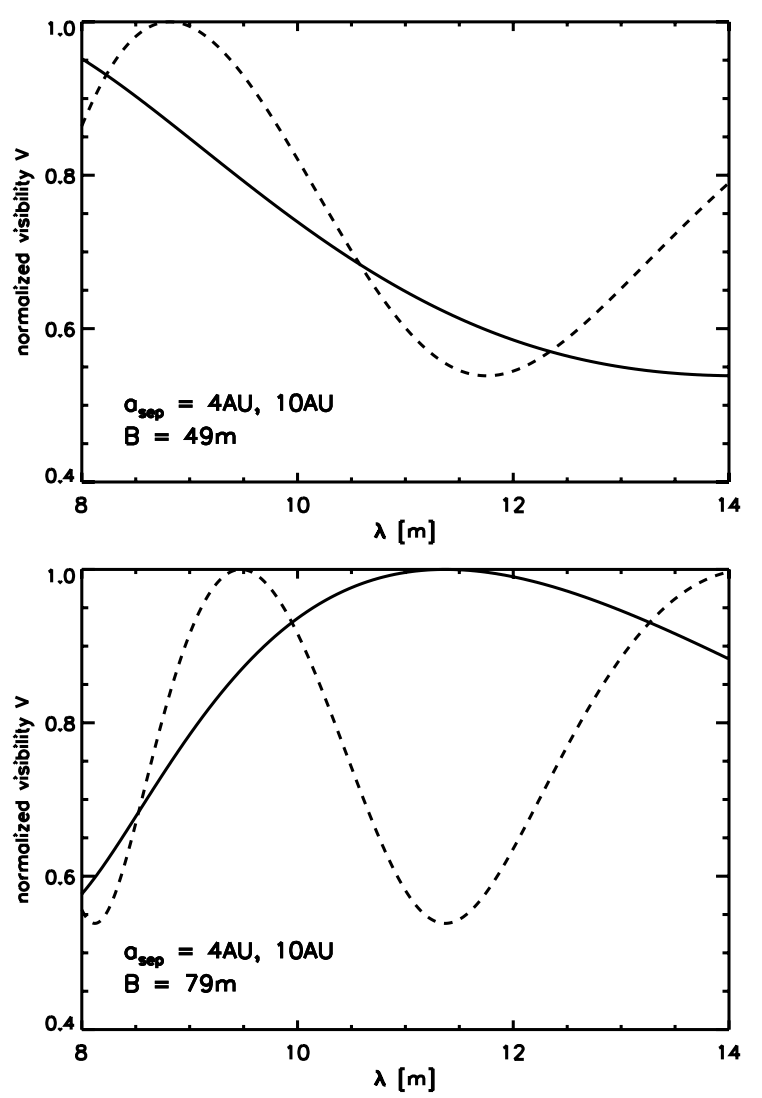

Fig. 9. Theoretical prediction for the result obtained for the observation of a stellar binary sytem with MIDI. Here, we assume two point sources, i.e. $a_{0}(B, \lambda)=1 \mathrm{in} \mathrm{Eq.} \mathrm{(8).} \mathrm{The} \mathrm{(normalized)} \mathrm{visibility} \mathrm{is} \mathrm{shown} \mathrm{in} \mathrm{rela-}$ tion with the separation of both stars (solid lines: $a_{\text {sep }}=4 \mathrm{AE}$; dashed lines: $a_{\text {sep }}=10 \mathrm{AE}$ ) and the projected baseline length (left: $B=49 \mathrm{~m}$; right: $B=79 \mathrm{~m})$. The brightness ratio of the components are $1: 3$. In this example the baseline of the interferometer is also parallel to the connection line of the components.

snapshots of the system in contrast to the HIPPARCOS observations repeated on long-term. A disadvantageous configuration of the secondary could actually prevent detection with the interferometer. With the non-dection of a clear binary signal in our MIDI data, we can thus neither verify, nor disprove the existence of a companion. Assuming $a_{0}(B, \lambda)=1$ for two point sources, Fig. 9 shows the relation between the interferometric binary signal and the projected baseline and the separation of the objects, respectively. With increasing baseline length or increasing separation the frequency of the sinusoidal variation also increases. The amplitude of the variation decreases with the brightness ratio of the components.

Another aspect of this discussion is the possibility that the regular motion of the photocenter observed with HIPPARCOS in the visible range is not caused by a secondary, but by a brightness irregularity in the circumstellar environment, i.e. in the disk and/or envelope. In fact, considering scattered-light images of Class I objects (Padgett et al. 1999), circumstellar envelopes, which have their brightness maximum in the NIR and adjacent wavelength ranges (Wolf et al. 2003), reveal such brightness irregularities. However, it is not clear if such a brightness irregularity in the circumstellar environment of RY Tau is strong enough to cause the observed regular motion of the photocenter. 


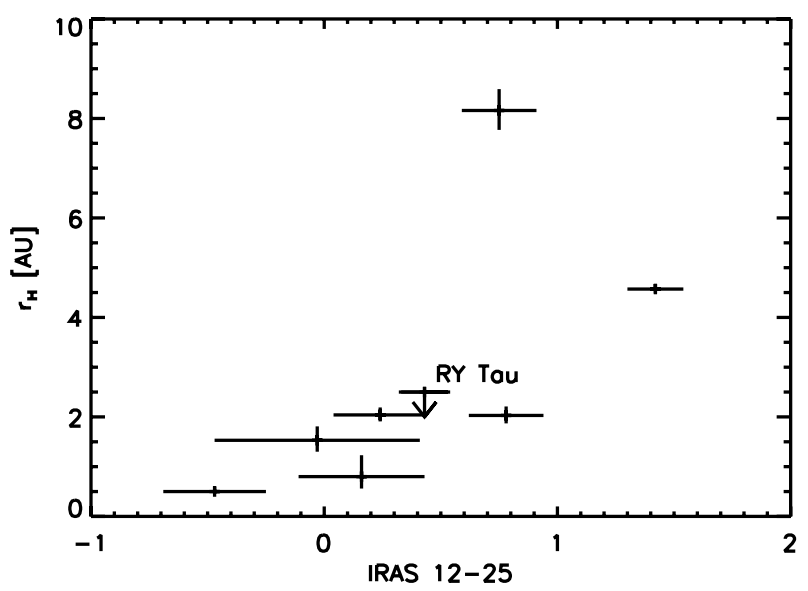

Fig. 10. Correlation between the half light radius $r_{\mathrm{H}}$ at a wavelength of $12.5 \mu \mathrm{m}$ and the IRAS-color at $\lambda=12 \mu \mathrm{m}$ and $\lambda=25 \mu \mathrm{m}$. Plotted are the corresponding values for HAeBe objects (Leinert et al. 2004) and for RY Tau. The error bars correspond to errors of the IRAS fluxes and of the interferometric measurements. The value $r_{\mathrm{H}}$ of RY Tau is an upper limit, as the active disk model predicts a too small visibility in comparison to the measurement.

\subsection{Comparison with $\mathrm{HAeBe}$ stars}

HAeBe stars were formerly classified in Group I and Group II sources (Meeus et al. 2001; Sect. 5.2). Recently, Leinert et al. (2004) could explain this phenomenological classification after modeling the interferometric measurements of several HAeBe objects obtained with MIDI. In fact, they noticed that the half light radius of their disk models in the MIR wavelength range $^{8}$ linearly correlates with the IRAS color between $12 \mu \mathrm{m}$ and $25 \mu \mathrm{m}$, i.e. $-2.5 \log \left(F_{v}(12 \mu \mathrm{m}) / F_{v}(25 \mu \mathrm{m})\right)$. Circumstellar disks around Group II sources are less flared. The geometrical effect results in the outer disk regions around Group II sources being less strongly heated than the corresponding disk regions of Group I sources. Therefore, the size of the half light radius and, simultaneously, the MIR color is smaller for Group II sources.

Because of its stellar luminosity $\left(L_{\star}=11.5 L_{\odot}\right)$ and stellar mass $\left(M_{\star}=1.69 M_{\odot}\right)$ the T Tauri star RY Tau could be considered as a transition object between T Tauri and HAeBe stars. As the FIR-flux declines, RY Tau can be classified as a Group II source (Meeus et al. 2001). The IRAS color of RY Tau in the MIR wavelength range is $-2.5 \log \left(F_{v}(12 \mu \mathrm{m}) / F_{v}(25 \mu \mathrm{m})\right)=$ $0,43 \pm 0,11$ (IRAS Catalogs 1985). The half light radius $r_{\mathrm{H}}$, i.e. the $F W H M$ of the intensity distribution in our best active disk model is determined here by a Gaussian fit. The intensity distribution is computed for a wavelength of $\lambda=12.5 \mu \mathrm{m}$ as it is not affected by the silicate emission at this wavelength. We obtain $F W H M=1.75$ AU. This value is an upper limit of the half light radius as the active disk model predicts a too small visibility in comparison to the measurement, i.e. the intensity distribution of the model disk decreases too slowly for increasing radii in contrast to the real intensity distribution (measurement). However, with respect to the study of Leinert et al. (2004, Fig. 5) this result actually confirms the linear correlation formerly found for HAeBe stars, solely. A further correspondence between T Tauri and HAeBe objects has been already shown in Schegerer et al. (2006) considering correlations between stellar properties and the silicate composition of the circumstellar disk of T Tauri and HAeBe objects.

8 The half light radius encircles the disk region emitting half of the totally released energy in the disk.

\subsection{Complex interplay of disk parameters}

The visibility, which we try to model, is a complex function of many different disk and dust parameters. In such complex disk models the effect of any modifications of geometrical disk parameters cannot always be predicted. However, the tendencies of modifications of the main model parameters are summarized in the following:

i. An increase of the stellar effective temperature $T_{\star}$ and stellar luminosity $L_{\star}$, in particular, mainly causes an increase of the visible and infrared flux. If the flux of the unresolved star increases more strongly than the heating of the dust and the MIR flux from the resolved circumstellar surroundings, the MIR-visibility increases. The accretion luminosity affects SED and MIR visibility, correspondingly. An increase of the stellar mass $M_{\star}$ corresponds to an increase of the accretion luminosity in the active disk model (Eq. (A.2)).

ii. The optical depth of the disk increases with the disk mass assuming a constant outer radius. Compacter disk structure decreases the averaged temperature in the inner disk region where less MIR flux is emitted. However, the MIR flux from outer regions simultaneously increases and the MIR visibility therefore decreases.

iii. A decrease of the inner disk radius $R_{\text {in }}$ results in more NIR and MIR flux and slightly increases the MIR visibility.

iv. As shown in Sect. 5.1 the outer disk radius determines the FIR flux in our modeling approach. The effect of an increase of the outer radius corresponds to the effect of a decrease of the disk mass.

v. Similar to the outer disk radius, the exponent $p$ has distinct effects on the radial density distribution in the disk. Surface density distributions $\Sigma$ with larger gradients $p$ result in an increase of the density in the inner regions of the disk. The MIR visibility and the infrared excess from the disk in the NIR up to the MIR wavelength range increases, simultaneously. While the FIR excess can decrease strongly at values greater than $p=2.0$, smaller exponents $p<0.8$ only negligibly affect the SED and MIR visibility.

The spatial information, which we got from the MIDI observations, strongly reduced the number of disk models which can reproduce the SED. Certainly, the outer disk radius and the disk mass are less constrained parameters in our models. The uniqueness of our approach cannot be proven. However, in our approach we consistently verified whether the modeling results can be improved by varying the modeling parameters. The step sizes used for a final variation of these modeling parameters are:

$\Delta R_{\text {out }}=10 \mathrm{AU}, \Delta R_{\text {in }}=0.05 \mathrm{AU}$,

$\Delta L=0.1 L_{\odot}, \Delta c_{2}=0.5$, and $\Delta p=0.1$.

For the parameter $c_{1}$ and $M_{\text {disk }}$ we used:

$\Delta c_{1}=0.1 c_{1}$, and $\Delta M_{\text {disk }}=0.5 M_{\text {disk }}$,

respectively. These step sizes can be considered as an error of each resulting parameter (Table 4). As mentioned above, the stellar mass $M_{\text {star }}$, the effective stellar temperature $T_{\star}$, the accretion rate $\dot{M}$, the boundary temperature $T_{\text {bnd }}$ and radius $R_{\text {bnd }}$ as well as the visual extinction $A_{\mathrm{V}}$ are constant parameters considering previous measurements.

\section{Summary}

We present interferometric observations of the classical T Tauri star RY Tau in the $10 \mu \mathrm{m}$ range, which show the source well 
resolved, together with the total spectrum of the source. We modified the MC3D code (Wolf et al. 1999) to obtain a selfconsistent model (in the temperature and density distribution) of the circumstellar disk around RY Tau, including accretion, with the following results:

i. Both, the SED and the $10 \mu \mathrm{m}$ interferometric measurements can be fitted by a self-consistent, physically reasonable radiative transfer model. The accretion and an envelope have the effect of filling in the missing emission in the range from $3 \mu \mathrm{m}$ up to $8 \mu \mathrm{m}$.

ii. A model of a circumstellar active disk with an optically thin envelope and a low accretion rate represents the data and the model without the envelope and a much larger accretion rate (Akeson et al. 2005). The modeling approach with an envelope, which could have its origin in an outflowing gaseous and dusty disk wind, should be considered as a future, viable alternative for the puffed-up inner rim, which results from a vertical expansion of the disk edge according to Natta et al. (2001) and Dullemond et al. (2001). The latter approach could not be confirmed with our particular model of RY Tau considering a self-consistently determined density distribution considering hydrostatical equilibrium.

iii. The interferometric observation reduces the number of models that solely reproduce the SED of RY Tau.

iv. The results of our modeling approaches are: The active disk with or without an (optically thin) envelope predicts a system with an upper limit of $\leqq 70^{\circ}$ for the inclination. While a stellar mass of $M_{\star}=1.69 M_{\odot}$ and an effective temperature of $5560 \mathrm{~K}$ can be adopted from previous measurements (Table 1), we found a stellar luminosity of $L_{\star} \approx 10 . L_{\odot}$ which is smaller than the estimates of Akeson et al. $\left(2005 ; 12.8 L_{\odot}\right)$ but corresponds to the findings of Calvet et al. (2004; $\left.9.6 L_{\odot}\right)$. The inner radius of our models $R_{\text {in }}=0.3 \mathrm{AU}$ coincides with the result of Akeson et al. $\left(2005 ; R_{\text {in }}=0.27 \mathrm{AU}\right)$ where interferometrical studies in the NIR preceded. The accretion rate of the model without an envelope is $9.1 \times 10^{-8} M_{\odot} \mathrm{yr}^{-1}$ and corresponds to the upper limit found by Calvet et al. (2004). The use of an additional envelope allows us to use a lower accretion rate of $2.5 \times 10^{-8} M_{\odot} \mathrm{yr}^{-1}$ which is the same as the result published by Hartigan et al. (1995).

v. Comparison of interferometric and single-dish observations shows for the first time dust evolution in a T Tauri star with a reduced fraction of small amorphous and an increased fraction of crystalline particles closer to the star. This is similar to the finding for HAeBe stars (van Boekel et al. 2004).

vi. Our data neither support nor contradict the existence of a close ( 3 AU) companion for which some astrometric evidence exists (Bertout et al. 1999).

vii. The complex interplay of different disk parameters is consistently apparent from the use of the Monte Carlo radiative transfer code.

Acknowledgements. A. A. Schegerer and S. Wolf were supported by the German Research Foundation (DFG) through the Emmy-Noether grant WO 857/2 ("The evolution of circumstellar dust disks to planetary systems").

\section{References}

Adams, F. C., Lada, C. J., \& Shu, F. H. 1987, ApJ, 312, 788 Akeson, R. L., Walker, C. H., Wood, K., et al. 2005, ApJ, 622, 440 Allen, L. E., Myers, P. C., Di Francesco, J., et al. 2002, ApJ, 566, 993 Andrews, S. M., \& Williams, J. P. 2006, [arXiv:astro-ph/0610813] Basri, G., Martin, E. L., \& Bertout, C. 1991, A\&A, 252, 625
Bastien, P, \& Landstreet, J. D. 1979, ApJ, 229, 137

Beckwith, S. V. W., Sargent, A. I., Chini, R. S., \& Güsten, R. 1990, AJ, 99, 924 Beckwith, S. V. W., Henning, Th., \& Nakagawa, Y. 1999, in Protostars and Planets IV, Dust Properties and Assembly of Large Particles in Protoplanetary Disks, ed. V. Mannings, A. P. Boss, \& S. S. Russell, 533

Bertout, C., Basri, G., \& Bouvier, J. 1988, ApJ, 330, 350

Bertout, C., Robichon, N., \& Arenou, F. 1999, A\&A, 352, 574

Blandford, R. D., \& Payne, D. G. 1982, MNRAS, 199, 883

Blum, J., \& Wurm, G. 2000, Icarus, 143, 128

Bohren, C. F., \& Huffman, D. R. 1983, in Absorption and scattering of light by small particles (New York: Wiley)

Boss, A. P. 2000, ApJ, 536, 101

Bouwman, J., Meeus, G., de Koter, A., et al. 2001, A\&A, 375, 950

Bouwman, J., de Koter, A., Dominik, C., \& Waters, L. B. F. M. 2003, 401, 577

Calvet, N., \& Gullbring, E. 1998, ApJ, 509, 802

Calvet, N., Hartmann, L., \& Strom, S. E. 1997, ApJ, 481, 912

Calvet, N., D'Alessio, P., Hartmann, L., et al. 2002, ApJ, 568, 1008

Calvet, N., Muzerolle, J., Briceño, C., et al. 2004, AJ, 128, 1294

Chiang, E. I., \& Goldreich, P. 1997, ApJ, 490, 368

Chiang, E. I., Joung, M. K., Creech-Eakman, M. J., et al. 2001, ApJ, 547, 1077

Cohen, M., \& Kuhi, L. V. 1979, ApJS, 41, 743

Cutri, R. M., Skrutskie, M. F., van Dyk, S., et al. 2003, 2MASS All-Sky Catalog of Point Sources

D’Alessio, P., Calvet, N., \& Hartmann, L. 1997, ApJ, 474, 397

D’Alessio, P., Cantó, J., Calvet, N., \& Lizano, S. 1998, ApJ, 500, 411

D’Alessio, P., Calvet, N., \& Hartmann, L. 2001, ApJ, 553, 321

Dominik, C., Dullemond, C. P., Waters, L. B. F. M., \& Walch, S. 2003, A\&A, 398, 607

Draine, B. T., \& Lee, H. M. 1984, ApJ, 285, 89

Draine, B. T., \& Malhotra, S. 1993, ApJ, 414, 632

Dullemond, C. P., Dominik, C., \& Natta, A. 2001, ApJ, 560, 967

Dullemond, C. P., \& Dominik, C. 2004, A\&A, 417, 159

Dullemond, C. P., \& Dominik, C. 2004, A\&A, 421, 1075

Dullemond, C. P., Hollenbach, D., Kamp, I., \& D’Alessio, P. 2006, in Protostars and Planets V, ed. B. Reipurth, D. Jewitt, \& K. Keil

Duschl, W. J., Gail, H.-P., \& Tscharnuter, W. M. 1996, A\&A, 312, 624

Edwards, S., Ray, T. P., \& Mundt, R. 1993, in Protostars and Planets III, Energetic Mass Outflows from Young Stars, ed. E. H. Levy, \& J. I. Lunine, 567

Elias, J. H. 1978, ApJ, 224, 857

Eiroa, C., Oudmaijer, R. D., Davies, J. K., et al. 2002, A\&A, 384, 1038

Eisner, J. A., Hillenbrand, L. A., White, R. J., Akeson, R. L., \& Sargent, A. I. 2005a, ApJ, 623, 952

Eisner, J. A., Hillenbrand, L. A., Carpenter, J. M., \& Wolf, S. 2005b, ApJ, 635, 396

Fendt, Ch., \& Camenzind, M. 1995, A\&A, 313, 591

Folha, D. F. M., \& Emerson, J. P. 1999, A\&A, 352, 517

Gail, H.-P. 1998, A\&A, 332, 1099

Gail, H.-P. 2003, in Astromineralogy, Formation and Evolution of Minerals in Accretion Disks and Stellar Outflows, LNP, ed. Th. Henning, 609, 55

Gail, H.-P. 2004, A\&A, 413, 571

Gezari, D. Y., Pitts, P. S., \& Schmitz, M. 1999, Catalog of Infrared Observations, edition 5

Hartmann, L. 1998, Accretion Processes in Star Formation, in Cambridge Astrophysics Series, ed. A. King, D. Lin, S. Maran, J. Pringle, \& M. Ward

Hartmann, L. 2005, in Astrophysical Observations of Disk Evolution around Solar Mass Stars, ed. A. N. Krot, E. R. D. Scott, \& B. Reipurth, ASP Conf. Ser., 341, 131

Hartmann, L., Kenyon, S. J., \& Calvet, N. 1993, ApJ, 407, 219

Hanner, M. S., Brooke, T. Y., \& Tokunaga, A. T. 1998, ApJ, 502, 871

Harvey, P. M., Thronson, H. A., \& Gatley, I. 1979, ApJ, 231, 115

Hartigan, P., Edwards, S., \& Ghandour, L. 1995, ApJ, 452, 736

Herbig, G. H., Petrov, P. P., \& Duemmler, R. 2003, ApJ, 595, 384

Herbst, W., \& Stine, P. C. 1984, AJ, 89, 1716

Herbst, W., \& Shevchenko, V. S. 1999, ApJ, 118, 1043

Indebetouw, R., Mathis, J. S., Babler, B. L., et al. 2005, ApJ, 619, 931

IRAS Explanatory Supplement to the Catalogs and Atlasses 1985, ed. C. Beichmann, G. Neugebauer, H. J. Habing, P. E. Clegg, \& T. J. Chester, NASA RP-1190, 1

Isella, A., \& Natta, A. 2005, A\&A, 438, 899

Jaffe, W. 2004, SPIE Proc., 5491, 715

Jäger, C., Mutschke, H., Begemann, B., Dorschner, J., \& Henning, Th. 1994, A\&A, 292, 641

Johansen, A., \& Klahr, H. 2005, ApJ, 634, 1353

Johns-Krull, C. M., Valenti, J. A., \& Gafford, A. D. 2003, Rev. Mex. Astron. Astrofis., 18, 38

Joy, A. H. 1945, ApJ, 102, 168

Kenyon, S. J., \& Hartmann, L. 1995, ApJS, 101, 117 
Klahr, H. 2004, ApJ, 606, 1070

Königl, A. 1991, ApJ, 370, 39

Kornet, K., Stepinski, T. F., \& Różyczka, M. 2001, A\&A, 371, 180

Kuhi, L. V. 1974, A\&AS, 15, 47

Kurucz, R. L. 1994, Kurucz CD-ROM 19, Solar Abundance Model Atmospheres, Cambridge, SAO

Laughlin, G., \& Bodenheimer, P. 1994, ApJ, 436, 335

Leinert, C., Zinnecker, H., Weitzel, N., et al. 1993, A\&A, 278, 129

Leinert, C., Bowyer, S., Haikala, L. K., et al. 1997, A\&AS, 127, 1

Leinert, C., Graser, U., Richichi, A., et al. 2003a, The Messenger, 112, 13

Leinert, C., Graser, U., Przygodda, F., et al. 2003b, A\&SS, 286, 73

Leinert, C., Graser, U., Waters, L. B. F. M., et al. 2003c, SPIE Proc., 4838, 893

Leinert, C., van Boekel, R., Waters, L. B. F. M., et al. 2004, A\&A, 423, 537

Lissauer, J. J. 1993, ARA\&A, 31, 129

Lodato, G., \& Bertin, G. 2001, A\&A, 375, 455

Lucas, P. W., \& Roche, P. F. 1997, MNRAS, 286, 895

Lynden-Bell, D., \& Pringle, J. E. 1974, MNRAS, 168, 603

Mannings, V., \& Sargent, A. I. 1997, ApJ, 490, 792

Mannings, V., \& Sargent, A. I., 2000, ApJ, 529, 391

Mathis, J. S., Rumpl, W., \& Nordsieck, K. H. 1977, ApJ, 217, 425

McCabe, C., Duchêne, G., \& Ghez, A. M. 2003, ApJ, 588, 113

Meeus, G., Waters, L. B. F. M., Bouwman, J., et al. 2001, A\&A, 365, 476

Millan-Gabet, R., Schloerb, F. P., \& Traub, W. 2001, ApJ, 546, 358

Millan-Gabet, R., Malbet, F., Akeson, R., et al. 2006, in Protostars and Planets V, ed. B. Reipurth, D. Jewitt, \& K. Keil

Miyake, K., \& Nakagawa, Y. 1995, ApJ, 441, 361

Monnier, J. D., Berger, J.-P., Millan-Gabet, R., et al. 2006, ApJ, 647, 444

Mora, A., Merin, B., \& Solano, E. 2001, A\&A, 378, 116

Muzerolle, J., D’Alessio, P., Calvet, N., \& Hartmann, L. 2004, ApJ, 617, 406

Nagasawa, M., Thommes, E. W., Kenyon, S. J., Bromley, B. C., \& Lin, D. N. C.

2006, in Protostars and Planets V, ed. B. Reipurth, D. Jewitt, \& K. Keil

Najita, T., Carr, J. S., \& Mathieu, R. D. 2003, ApJ, 589, 931

Nakajima, T., \& Golimowski, D. A. 1995, AJ, 109, 1181

Natta, A., Meyer, M. R., \& Beckwith, S. V. 2000, ApJ, 534, 838

Natta, A., Prusti, T., Neri, R., et al. 2001, A\&A, 371, 186

Padgett, D. L., Brandner, W., Stapelfeldt, K. R., et al. 1999, AJ, 117, 1490

Pascucci, I., Wolf, S., Steinacker, J., et al. 2004, A\&A, 417, 793

Pavlyuchenkov, Ya., \& Dullemond, C. P. 2007, A\&A, 471, 833

Perryman, M. A. C., ESA 1997, in The Hipparcos and Tycho catalogues, ESA SP Ser., 1200

Petrov, P. P., Zajtseva, G. V., Efimov, Yu. S., et al. 1999, A\&A, 341, 553

Pickering, E. C. 1907, AN, 175, 333
Pontoppidan, K. M., Dullemond, C. P., van Dishoeck, E. F., et al. 2005, ApJ, 622,463

Preibisch, Th., Kraus, St., Driebe, Th., van Boekel, R., \& Weigelt, G. 2006, A\&A, 458, 235

Pringle, J. E. 1981, ARA\&A, 19, 137

Ratzka, T. 2005, Ph.D. Thesis, Ruprecht-Karls-Universität Heidelberg

Richer, J. S., Shephard, D. S., Cabrit, S., Bachiller, R., \& Churchwell, E. 2000, in Protostars and Planets IV, ed. V. Mannings, A. Boss, \& S. S. Russell

Rodmann, J., Henning, Th., Chandler, C. J., Mundy, L. G., \& Wilner, D. J. 2006, A\&A, 446, 211

Rydgren, A. E., Strom, S. E., \& Strom, K. M. 1976, ApJS, 30, 307

Schegerer, A., Wolf, S., Voshchinnikov, N. V., Przygodda, F., \& Kessler-Silacci, J. E. 2006, A\&A, 456, 535

Siess, L., Forestini, M., \& Bertout, C. 1999, A\&A, 342, 480

Sonnhalter, C., Preibisch, Th., \& Yorke, H. W. 1995, A\&A, 299, 545

Strom, K. M., Strom, S. E., Edwards, S., Cabrit, S., \& Skrutsksie, M. F. 1989, AJ, 97, 1451

Uchida, Y., \& Shibata, K. 1984, PASJ, 36, 105

Ulrich, R. K. 1976, ApJ, 210, 377

van Boekel, R., Min, M., Leinert, Ch., et al. 2004, Nature, 432, 479

Vink, J. S., Drew, J. E., Harries, T. J., Oudmaijer, R. D., \& Unruh, Y. C. 2003, A\&A, 406, 703

Vinković, D., Ivezić, Z., Jurkić, T., \& Elitzur, M. 2006, ApJ, 636, 348

Wehrstedt, M., \& Gail, H.-P. 2002, A\&A, 385, 181

Weidenschilling, S. J. 1977, APSS, 51, 153

Weidenschilling, S. J. 1997, in From Stardust to Planetesimals, ed. Y. J. Pendleton, \& A. G. G. M. Tielens, ASP Conf. Ser., 122, 281

Weinberger, A. J., Becklin, E. E., Schneider, G., et al. 1999, ApJ, 525, 53

Weingartner, J. C., \& Draine, B. T. 2001, ApJ, 548, 296

Wielen, R. 1996, A\&A, 314, 679

Whitney, B. A., Wood, K., Bjorkman, J. E., \& Cohen, M. 2003, ApJ, 598, 1079

Wolf, S. 2003a, ApJ, 582, 859

Wolf, S., \& Hillenbrand, L. A. 2003b, ApJ, 596, 603

Wolf, S., Henning, Th., \& Stecklum, B. 1999, A\&A, 349, 839

Wolf, S., Padgett, D. L., \& Stapelfeldt, K. R. 2003c, ApJ, 588, 373

Wood, K., Lada, C.J., Bjorkman, J.E., et al. 2002b, ApJ, 567, 1183

Wünsch, R., Klahr, H., \& Rózyczka, M. 2003, MNRAS, 362, 361

Wünsch, R., Klahr, H., \& Różycka, M., 2005, MNRAS, 362, 361

Wurm, G. 2005, LPI Contr., 1280, 161

Wurm, G., \& Krauss, O. 2006, Phys. Rev. Lett., 96, 4301

Yorke, H. W., Bodenheimer, P., \& Laughlin, G. 1993, ApJ, 411, 274

Zajtseva, G., Kolotilov, E. A., Petrov, P. P., et al. 1985, SvA Lett. 11, 109 
A. A. Schegerer et al.: The inner regions of circumstellar dust disks, Online Material $p 1$

\section{Online Material}




\section{Appendix A: The accretion model}

Accretion models have been studied in many publications and the parameters that we used for our active disk model are wellknown properties of such models. However, only a few authors have implemented accretion effects in radiative transfer models (e.g., Akeson et al. 2005). It is generally assumed that the passive heating of the disk is the dominating source of infrared and $\mathrm{mm}$ irradiation. This is certainly true for the large scales of the disk but not for its innermost region ( $<2$ AU; e.g., D'Alessio et al. 1998) which can be resolved with long-baseline interferometers. We briefly summarize here the parameters of the accretion disk model implemented in our approach.

Former accretion disk models are generally based on the assumption of a geometrically thin, steady disk established by Lynden-Bell \& Pringel (1974) and Pringle (1981). In this canonical accretion model it is assumed that viscous stresses within the disk transport angular momentum to its outer regions. As a consequence of this, most of the disk material moves inward onto the protostar, while some disk matter moves outward, absorbing all the angular momentum. Assuming a geometrically thin and steady disk the conservation of transversal and angular momentum results in the dissipation rate $D$ per unit area and time as a function of the radial distance from the star $r$ :

$D(r)=\frac{3 G M_{\star} \dot{M}}{4 \pi r^{3}}\left[1-\left(\frac{R_{\star}}{r}\right)^{1 / 2}\right]$.

The quantities $G, M_{\star}, \dot{M}, r$ and $R_{\star}$ are in this order: gravity constant, stellar mass, accretion rate of the infalling material, radial distance and stellar radius. The total released energy between an inner boundary radius of the disk $R_{\text {bnd }}$ and infinity is

$L_{\mathrm{disk}}=\int_{R_{\mathrm{bnd}}}^{\infty} D(r) 2 \pi r \mathrm{~d} r=\frac{1}{2} \frac{G M_{\star} \dot{M}}{R_{\mathrm{bnd}}}$.

Lynden-Bell \& Pringle (1974) assumed that the disk extends down to the stellar radius $R_{\text {bnd }}=R_{\star}$, or to the corotation radius where the Keplerian orbital period equals the stellar rotation period. Recent observations (e.g., Muzerolle et al. 2004) confirmed a magnetically mediated accretion processes in the innermost disk. The accretion disk is truncated by the stellar magnetic field at a radius $R_{\text {bnd }}$ of several stellar radii and material is channeled along magnetic field lines onto the star with nearly free-fall velocity (e.g., Uchida \& Shibata 1984; Bertout et al. 1988). The magnetic flux density $B$ of a T Tauri star is in the range of $\sim 1-$ $3 \mathrm{kG}$ (e.g., Johns-Krull et al. 2003). As the boundary radius $R_{\text {bnd }}$ is assumed to be smaller than the sublimation radius of dust $\left(\approx R_{\text {in }}\right.$ for RY Tau), the accreting material in this inner region is gaseous. Close to the stellar surface the accreting, freefalling material is abruptly stopped in one or a series of shock layers. In this inner accretion zone the total released energy is $\zeta G M_{\star} \dot{M} / R_{\star}$ with $\zeta=1-R_{\star} /\left(2 R_{\text {bnd }}\right)$. Therefore, adjacent layers of the shock region like the stellar photosphere are heated to temperatures of $T_{\text {bnd }}=5700 \mathrm{~K}$ up to $8800 \mathrm{~K}$, while temperatures of 9000 up to $20000 \mathrm{~K}$ are achieved in upper shock areas (Calvet \& Gullbring 1998). Muzerolle et al. (2004) found in their studies that the energy contribution from the upper shock layers can be neglected for accretion rates $\dot{M} \leqq 10^{-6} M_{\odot} \mathrm{yr}^{-1}$ while the stellar photosphere emits most of the accretion energy. Thus, the stellar photosphere is assumed to emit the intrinsic stellar flux and the accretion energy $\zeta G M_{\star} \dot{M} / R_{\star}$.

The crux of the accretion theory is the surface coverage factor $f$ which mimics an annulus on the stellar photosphere where the additional accretion emission occurs (D'Alessio et al. 1998; Bertout et al. 1988; Lynden-Bell \& Pringle 1974). If a blackbody emitter is assumed, one gets:

$$
f 4 \pi R_{\star}^{2} \sigma T_{\text {bnd }}^{4}=\zeta G M_{\star} \dot{M} / R_{\star} .
$$

The Stefan-Boltzmann constant is represented by $\sigma$. Calvet \& Gullbring (1998) determined $f \approx 0.1-1.0 \%$ for HAeBe stars and $f \approx 10 \%$ for T Tauri stars. Stronger veiling of absorption lines indicates the larger coverage factors $f$ of T Tauri stars.

In the region between the sublimation and magnetic boundary radius the gradually released accretion rate in our model (see Eq. (A.1)) is determined by the accretion theory of Lynden-Bell $\&$ Pringles (1974). Because of the steep decrease of the dissipation rate $D$ with increasing distance $r$ (see Eq. (A.1)) accretion at radial distances $r>R_{\text {in }}$ is neglected in our model. The remaining gravitational energy of the accreting material between $r=R_{\text {bnd }}$ and $r=R_{\star}$ is emitted at the stellar surface assuming a blackbody emitter with the temperature $T_{\text {bnd }}$. Note that the effects of the boundary temperatur $T_{\text {bnd }}$ and the magnetic truncation radius $R_{\text {bnd }}$ on the SED and MIR visibilities are marginal (see D'Alessio et al. 1998). A sketch of our active disk model is shown in Fig. A.1. 
A. A. Schegerer et al.: The inner regions of circumstellar dust disks, Online Material $p 3$

gas \& disk disk
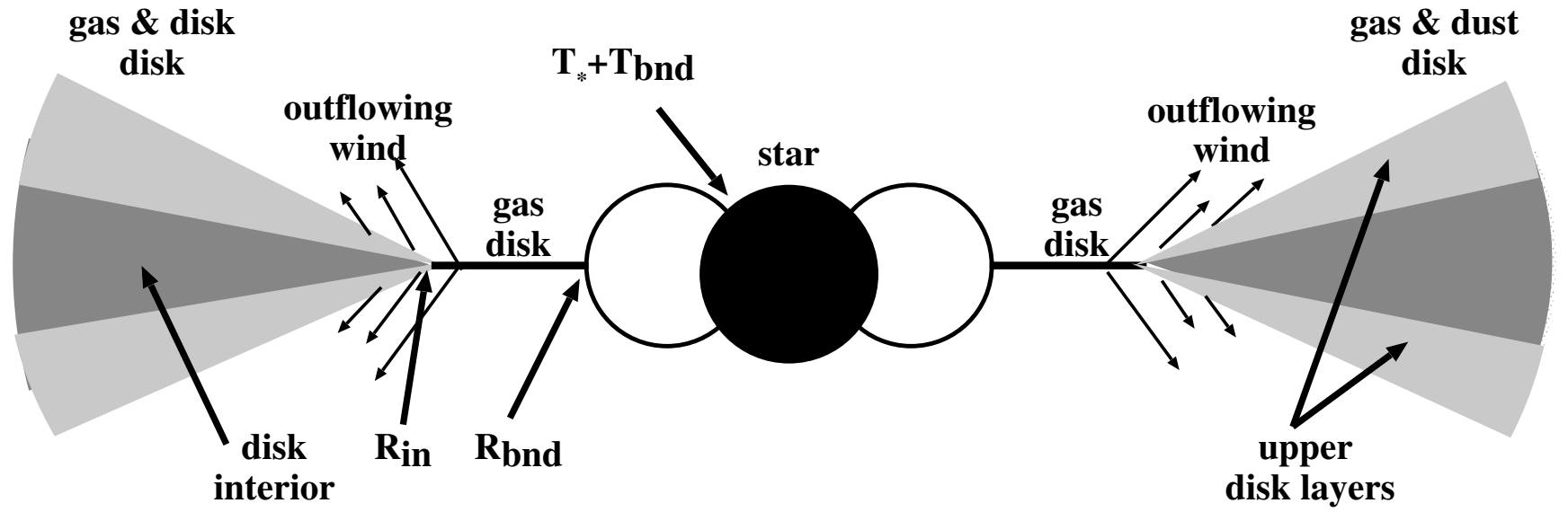

Fig. A.1. Simplified sketch of our active disk model. The boundary temperature $T_{\mathrm{bnd}}$, the boundary radius $R_{\mathrm{bnd}}$ and the inner disk radius $R_{\text {in }}$ are shown. We use a two-layer disk model (Sect. 4.2): a disk atmosphere where the optical depth $\tau_{\mathrm{N}}$ is smaller than unity in $N$ band and a optical thick, disk interior. 